\title{
Sensitivity analysis by design of experiments
}

An Van Schepdael, Aurélie Carlier and Liesbet Geris

\begin{abstract}
The design of experiments (DOE) is a valuable method for studying the influence of one or more factors on the outcome of computer experiments. There is no limit to the number of times a computer experiment can be run, but they are often time-consuming. Moreover, the number of parameters in a computer model is often very large and the range of variation for each of these parameters is often quite extensive. The DOE provides the statistical tools necessary for choosing a minimum amount of parameter combinations resulting in as much information as possible about the computer model. In this chapter, several designs and analysing methods are explained. At the end of the chapter, these designs and methods are applied to a mechanobiological model describing tooth movement.
\end{abstract}

Key words: design of experiments, sampling parameter space, sensitivity analysis

\section{Introduction}

The design of experiments (DOE) is a valuable method for studying the influence of one or more factors on physical experiments (see tutorial [19]). Physical experiments can often only be run a limited number of times and can be expensive and time-consuming. Therefore, when performing a sensitivity analysis on a model with

An Van Schepdael

Biomechanics Research Unit, University of Liège, Chemin des Chevreuils 1 - BAT 52/3, 4000

Liège, Belgium e-mail: An.VanSchepdael@mech.kuleuven.be

Aurélie Carlier

Biomechanics Section, University of Leuven, Celestijnenlaan 300C, box 2419, 3001 Heverlee, Belgium e-mail: Aurelie.Carlier@mech.kuleuven.be

Liesbet Geris

Biomechanics Research Unit, University of Liège, Chemin des Chevreuils 1 - BAT 52/3, 4000

Liège, Belgium e-mail: Liesbet.Geris@ulg.ac.be 
many parameters, limiting the number of parameter combinations to be studied is very important. The basic problem of designing such experiments is deciding which factor combinations to examine. The design of experiments (DOE) - introduced by Fisher [6] - was developed for this purpose.

There is no limit to the number of times a computer experiment can be run, but they are often time-consuming. Moreover, the number of parameters in a computer model is often very large and the range of variation for each of these parameters is often larger than in physical experiments. Although there are fundamental differences between physical experiments and computer simulations, the techniques of DOE that were originally developed for physical experimentation can also be used to investigate the sensitivity of a computer model to its parameters with a minimum of computational time.

\section{Theory}

Running a sensitivity analysis of a computer model using the DOE consists of three steps. Firstly, a suitable design, meaning a number of parameter combinations for which we will run the model, has to be set up. The purpose of this design is to get as much information as possible about the influence of the relevant parameters on the outcome of the model at minimal cost. In computer models, this cost is usually the computational time, which is kept low by limiting the number of parameter combinations that is studied. Next, simulations are run with these parameter combinations and finally, the results are analysed and conclusions are drawn [23, 24].

\subsection{Available designs}

A number of designs are available to conduct a sensitivity analysis [23, 24, 31]. This section provides an overview of the different techniques that are most commonly found in the biomedical literature.

\subsubsection{OAT-design}

The simplest design is a one-at-a-time (OAT) analysis, where each parameter is varied individually. A standard OAT-design uses a reference condition and then changes each parameter individually to a higher and a lower value, while keeping other parameters at the reference value. The difference between the outcome for the high and the low value is then used as a measure of the influence of the parameter on the system. The main advantage of this design is its simplicity and the fact that it only requires $2 M$ experiments, with $M$ being the number of parameters studied. It is however impossible to study interactions between parameters, and the effect of the 
parameters resulting from this analysis might be different when choosing a different reference condition [12]. The OAT analysis was used by Lacroix [17] and Geris et al. [8] to assess the influence of the value and duration of the initial and boundary conditions on the simulation results of a fracture healing model.

\subsubsection{Factorial designs}

In factorial designs, the parameters are assigned different values. In two-level designs, two different levels, a high and a low level, are chosen. Several combinations of parameter values are then compared, changing various parameters at the same time. In a two-level full factorial design, all possible combinations are examined, requiring $2^{M}$ experiments (see figure 1a). In three-level designs, requiring $3^{M}$ runs, the outcome of the model is also studied with parameters at an intermediate level (figure 1b). The advantage is that the effect of each parameter can be studied, and that interactions between the factors can be examined. Furthermore, no reference condition is required, giving the results more reliability. The main disadvantage is the computational cost $[23,31]$. The design requires $2^{M}$ runs, which becomes very high when the model contains many parameters. With 30 parameters, this would require $1.07 \times 10^{9}$ runs.

In fractional factorial designs, not all of these $2^{M}$ or $3^{M}$ combinations are examined (figure 1c). In a two-level full fractional factorial design with six parameters, only six out of 64 runs are used to estimate the main effects, and 15 are used to estimate the two-factor interactions. The remaining runs are used to calculate higher order interactions [24]. Fractional factorial designs are based on the principle that, most likely, at some point the higher order interactions become negligible. It is thus not necessary to examine all possible combinations of parameters, but it is sufficient to choose a suitable set of combinations [24, 31]. By omitting several combinations compared to the full factorial design, the amount of information gained from the sensitivity analysis decreases. The number of runs remains however limited resulting in a significant computational gain. Depending upon the number of experiments, several interactions will become indistinguishable. When using a minimum amount of experiments, only the effect of each parameter separately, the main effects, can be determined. When increasing the number of runs, two-factor interactions can be examined. Generally speaking, few experiments worry about higher order interactions. Fractional factorial designs are classified according to the level of information they provide. A resolution III design is set up in such way that the main effects are distinguishable, but may be affected by one or more two-factor interactions. These must thus assumed to be zero in order for the results to be meaningful. In a resolution IV design, the main effects can be distinguished from the other main effects and the two-factor interactions, but the two-factor interactions are confounded with each other [24, 23, 31]. A fractional factorial design is thus a trade-off between computational cost and accuracy, and are most frequently used to identify a subset of parameters that is most important and needs to be studied more extensively [24]. 
The main disadvantage is that the parameters are only studied at several levels and the values are not spread out over the entire parameter space.

Several other factorial designs are possible; Plackett-Burman designs, Cotter designs and mixed-level designs offer alternatives to standard fractional factorial designs, each having its own specific advantages and disadvantages. Isaksson et al. [11] determined, for example the most important cellular characteristics for fracture healing using a resolution IV fractional factorial design. Such design was also used by Malandrino et al. [21] to analyse the influence of six material properties on the displacement, fluid pore pressure and velocity fields in the L3-L4 lumbar intervertebral disc.

\subsubsection{Taguchi's design}

Taguchi's design was originally developed to assist in quality improvement during the development of a product or process. In a manufacturing process, for example, there are control factors and noise factors. The latter cause a variability in the final products and are usually uncontrollable. The goal of robust parameter design is the find the levels of the control factors that are least influenced by the noise factors [23]. In the Taguchi parameter design methodology one orthogonal design is chosen for the control factors (inner array) and one design is selected for the noise factors (outer array).

Taguchi's methodology has received a lot of attention in statistical literature. His philosophy was very original, but the implementation and technical nature of data analysis has received some criticism. Firstly, it does not allow the estimation of interaction terms. Secondly, some of the designs are empirically determined, but are suboptimal compared to rigorous alternatives such as fractional factorial designs [23]. Finally, if the Taguchi approach works and yields good results, it is still not clear what caused the result because of the aliasing of critical interactions. In other words, the problem may be solved short-term, without gaining any long-term process knowledge. Despite this criticism, Taguchi's approach is often used in biomedical literature because of its simplicity $[2,18,38]$.

\subsubsection{Space-filling designs}

In space-filling designs, the parameter combinations are spread out over the entire parameter space, enabling the design to capture more complex behaviour [33]. This approach is particularly useful for deterministic or near deterministic systems, such as computer simulations. To achieve an effective spreading of the parameters, several sampling methods are available. One of the most used methods is latin hypercube sampling (LHD). This method can be most easily explained by using the very simple example of a $2 \mathrm{D}$ experimental region, representing a system with 2 parameters $x_{1}$ and $x_{2}$ (figure 1d). For a design with $N$ runs, the region is divided into $N$ equally spaced rows and columns, creating $N^{2}$ cells. The points are then spread out, 
so that each row and column contains exactly one point. The main advantage of this method is that a latin hypercube design is computationally cheap to generate and that it can deal with a high number of parameters $[33,5]$. The main disadvantage however, is that the design is not flexible with regard to adding or excluding runs. By changing the number of runs, the condition that each row and column contains exactly one point is no longer met. Furthermore, LHD is well suited for monotonic functions, but might not be adequate for other systems [5].Finally, LHD designs are not necessarily space-filling (figure 1e). More elaborate algorithms which aim at ensuring the space-filling property of latin-hypercube designs, are described by Fang et al. [5].

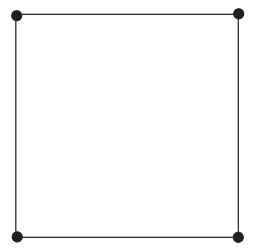

(a)

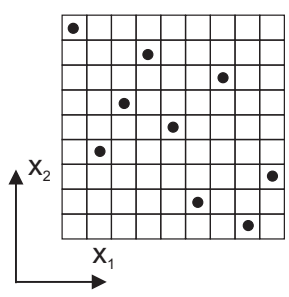

(d)

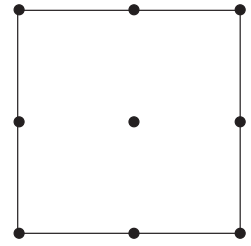

(b)

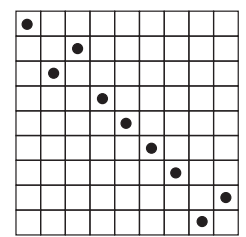

(e)

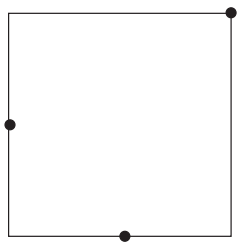

(c)

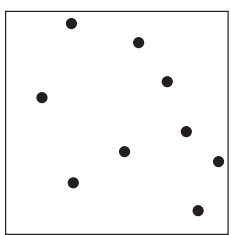

(f)

Fig. 1: Schematical overview of different designs for two factors $x_{1}$ and $x_{2}$. a A two-level full factorial design. b A three-level full factorial design. c A three-level fractional factorial design. d A latin hypercube design with nine runs. The parameter space is divided into $9^{2}=81$ cells, and one cell on each row and column is chosen. e A latin hypercube design with nine runs. This example shows that a LHD design is not necessarily space-filling. f A uniform design. Note that the factorial designs used discrete values of the parameters, while the LHD and uniform designs spread out the points in space.

Another method to achieve an effective spreading in space-filling designs is uniform sampling [4, 5]. In uniform designs, the parameters are spread out over space as uniformly as possible (figure 1f). The higher the number of runs, the better the 
spreading will be. Uniform designs are found to be efficient and robust, easy to understand and convenient, but computationally very demanding. Although this is a disadvantage, the fact that uniform designs cope well with the adding and removing of parameter combinations to the design makes them very useful in biomedical applications. For example, Carlier et al. [1] used a latin hypercube and uniform design to determine the most influential parameters of a calcium model that describes the effect of $\mathrm{CaP}$ biomaterials on the activity of osteogenic cells.

\subsection{Methods for analysing the results of a design}

Once a suitable design has been set up and computer simulations are run with the different parameter combinations, the results have to be analysed. Depending on the design and the goal of the analysis several methods are available. Analysis of variance (ANOVA) is particularly suited for analysing the outcome of a (full or fractional) factorial design, giving an indication of the importance of the investigated parameters. For the more complex space-filling designs, Gaussian processes are more appropriate, as they not only determine the importance of a parameter but also giving an estimate of the exact effect of varying a particular parameter on the outcome of the model. That way, more complex and non-linear effects can be revealed.

\subsubsection{Analysis of variance (ANOVA)}

Analysis of variance (ANOVA) can be used to investigate the result of a full or fractional factorial design. Firstly, the total variation in the output is modelled by calculating the total sum of squares of the deviation about the mean $\left(S S_{T}\right)$ [12].

$$
S S_{T}=\sum_{i=1}^{N}\left[y_{i}-\bar{y}\right]^{2}
$$

In this equation, $N$ is the number of runs, $y_{i}$ the output for the $i$ th run, and $\bar{y}$ the overall mean of the output. The influence of one parameter is determined by $S S_{F}$ :

$$
S S_{F}=\sum_{i=1}^{L} N_{F, i}\left[\bar{y}_{F, i}-\bar{y}\right]^{2},
$$

where $L$ is the number of levels used for each parameter, $N_{F, i}$ is the number of runs at each level of each factor and $\bar{y}_{F, i}$ is the mean output at each level of each factor. The percentage of the total sum of square,

$$
\% \text { TSS }=\left[S S_{F} / S S_{T}\right] \times 100 \%
$$


is a measure of importance for the parameter to the defined outcome [2].

\subsubsection{Gaussian process}

Gaussian processes not only estimate the importance of individual parameters, but also the influence of the parameters on the outcome of a model. Given the output data $\mathbf{t}_{N}=\left\{t_{i}\right\}_{i=1}^{N}$ resulting from a combination $\mathbf{X}_{N}=\left\{\mathbf{x}_{i}\right\}_{i=1}^{N}$ of input parameters, determined in the set-up of the design, Gaussian processes are used to predict the output $t_{*}$ for a certain combination $\mathbf{x}_{*}$ of input parameters [20]. To make this prediction, the output data are studied (figure 2), and a function $y(\mathbf{x})$ is searched, so that $y\left(\mathbf{x}_{i}\right)$ approaches the measured data $t_{i}$ as closely as possible. In linear regression, the function $y(\mathbf{x})$ is assumed to be linear and usually least square methods are applied to find the most likely result for $y(\mathbf{x})$. This analysis method however implies that assumptions have to be made regarding the form of the function, prior to analysing the data.

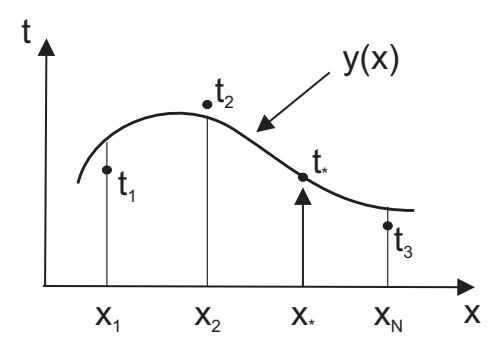

Fig. 2: Schematic representation of a Gaussian process on a system with output $t$, depending on one parameter $x$. The system is analysed for parameter values $\left\{x_{1}, x_{2}, \ldots, x_{N}\right\}$, for which output values $\left\{t_{1}, t_{2}, \ldots, t_{N}\right\}$ are obtained. In order to find the output value $t^{*}$ resulting from parameter value $x^{*}$, the Gaussian process searches for a function $y(x)$ which can explain the output values $\left\{t_{1}, t_{2}, \ldots, t_{N}\right\}$ the best. $t^{*}$ is then found as $t^{*}=y\left(x^{*}\right)$.

A Gaussian process starts from the following posterior probability function:

$$
P\left(y(\mathbf{x}) \mid \mathbf{t}_{N}, \mathbf{X}_{N}\right)=\frac{P\left(\mathbf{t}_{N} \mid y(\mathbf{x}), \mathbf{X}_{N}\right) P(y(\mathbf{x}))}{P\left(\mathbf{t}_{N} \mid \mathbf{X}_{N}\right)} .
$$

The first factor on the right hand side of (4), $P\left(\mathbf{t}_{N} \mid y(\mathbf{x}), \mathbf{X}_{N}\right)$, is the probability of the measured data given the function $y(\mathbf{x})$, and the second factor $P(y(\mathbf{x}))$ is the prior distribution on functions assumed by the model. In linear regression, this prior specifies the form of the function (e.g.: $y=a x+b$ ), and might put some restrictions on the parameters (e.g.: $a \neq 0$ ). The idea of Gaussian process modelling is to place a prior $P(y(\mathbf{x}))$ directly on the space of functions, without making assumptions on the form of the function. Just as a Gaussian distribution is fully defined by the mean 
and the covariance matrix, a Gaussian process is defined by a mean function and a covariance function. The mean is thus a function $\mu(\mathbf{x})$, which is often assumed to be the zero function, and the covariance is a function $\mathbf{C}\left(\mathbf{x}, \mathbf{x}^{\prime}\right)$. The only restriction on the covariance function is that it must be positive semi-definite. Several functions have been used widely, and proven valuable in literature. To get a better grasp on what exactly the covariance function represents and how a choice between the different available functions has to be made, an intuitive approach to developing the covariance function is explained below. A fully detailed and more theoretical approach can be found in Mackay [20].

Consider a system dependent upon one parameter $x$, with $N$ parameter values $x_{i}$, and a parametrisation of $y(x)$ using a set of basis functions $\left\{\phi_{h}(x)\right\}_{h=1}^{H}$. The function $y(x)$ can then be written as:

$$
y(x, \mathbf{w})=\sum_{h=1}^{H} w_{h} \phi_{h}(x) .
$$

As basis functions $\left\{\phi_{h}(x)\right\}$, radial basis functions centred at fixed points $\left\{c_{h}\right\}$ are chosen (figure 3).

$$
\phi_{h}(x)=e^{-\frac{\left[x-c_{h}\right]^{2}}{2 r^{2}}}
$$

Using the input points $\left\{x_{i}\right\}$ and the $H$ basis functions $\phi_{h}$, an $N \times H$ matrix $\mathbf{R}$ can be defined.

$$
R_{i h}=\phi_{h}\left(x_{i}\right)
$$

For a certain set of parameters $\mathbf{w}$, the function $y(x)$ then has the values $\mathbf{y}_{N}=\left\{y_{i}\right\}$ at the input points $x_{i}$.

$$
y_{i}=\sum_{h=1}^{H} w_{h} \phi_{h}\left(x_{i}\right)=\sum_{h=1}^{H} w_{h} R_{i h}
$$

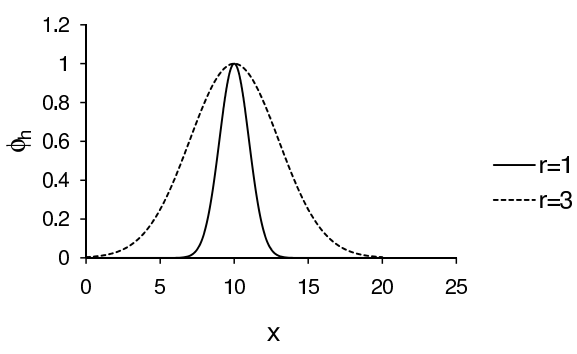

Fig. 3: Radial basis functions centred at $c_{h}=10$, used for the parametrisation of $y(x)$. The parameter $r$ is a length scale defining the width of the basis function. A larger value of $r$ results in a wider basis function and a smoother approximation of the function $y(x)$. 
In parametric regression methods, it is normally assumed that the prior distribution of the parameters $\mathbf{w}$ is Gaussian with a zero mean.

$$
P(\mathbf{w}) \sim \mathscr{N}\left(0, \sigma_{w}^{2} \mathbf{I}\right)
$$

In that case, $\mathbf{y}_{N}$, being a linear function of $\mathbf{w}$ is also Gaussian distributed with mean zero and covariance matrix $\mathbf{Q}$.

$$
\mathbf{Q}=\left\langle\mathbf{y}_{N} \mathbf{y}_{N}^{T}\right\rangle=\sigma_{w}^{2} \mathbf{R R}^{T}
$$

If each data point $t_{n}$ is assumed to differ by additive Gaussian noise of variance $\sigma_{v}^{2}$ from the corresponding function value $y\left(x_{i}\right)$, then:

$$
\begin{aligned}
P(\mathbf{t}) & \sim \mathscr{N}\left(0, \mathbf{Q}+\sigma_{v}^{2} \mathbf{I}\right) \\
& \sim \mathscr{N}(0, \mathbf{C}) .
\end{aligned}
$$

Now the assumption is made that the radial basis functions are uniformly spaced, that $H \rightarrow \infty$ and that $\sigma_{w}^{2}=S /(\Delta H)$, where $\Delta H$ is the number of base functions per unit length of the x-axis. The summation over $h$ then becomes an integral and the $(i, j)$ entry of $\mathbf{Q}$ equals:

$$
Q_{i j}=\sqrt{\pi r^{2}} S e^{-\frac{\left[x_{j}-x_{i}\right]^{2}}{4 r^{2}}}
$$

The covariance function $\mathbf{C}\left(\mathbf{x}, \mathbf{x}^{\prime}\right)$ of the Gaussian process is thus related to the basis functions chosen in the model. The parameter $r$ is a length parameter describing the width of the basis function. For a high value of $r$, the basis functions are wider, implying a higher correlation of the values of $y(x)$ at input points $x_{i}$ and $x_{j}$, resulting in a smoother function.

As mentioned before, several forms of the covariance function are possible. The first one used is the Gaussian or squared exponential covariance function.

$$
Q_{i j}=\sigma^{2} e^{-\sum_{m=1}^{M} \theta_{m}\left[x_{i m}-x_{j m}\right]^{2}}=\sigma^{2} \prod_{m=1}^{M} e^{\theta_{m}\left[x_{i m}-x_{j m}\right]^{2}}
$$

The summation in (13) is a result of the $M$-dimensional nature of the parameter combinations, which has not been taken into account in the intuitive approach, but is reintroduced here. The parameter $1 / \theta_{m}$, corresponding to parameter $m$, is related to the length scale $r$ described above. A very large number of $\theta_{m}$ implies a short length scale, indicating the function value will change significantly when changing the parameter. A value of $\theta_{m}=0$ implies an infinite length scale, meaning $y$ is a constant function of that input.

The second form for the covariance function is the cubic correlation covariance function. The covariance matrix is composed of the following elements:

$$
Q_{i j}=\sigma^{2} \prod_{m=1}^{M} \rho\left(d_{i j m} \theta_{m}\right)
$$


where

$$
\begin{aligned}
d_{i j m} & =x_{i m}-x_{j m} \\
\rho(d \theta) & =\left\{\begin{array}{lr}
1-6[d \theta]^{2}+6[|d| \theta]^{3}, & |d| \leq \frac{1}{2 \theta} \\
2[1-|d| \theta]^{3}, & \frac{1}{2 \theta} \leq|d| \leq \frac{1}{\theta} \\
0, & \frac{1}{\theta} \leq|d| .
\end{array}\right.
\end{aligned}
$$

Equation (16) shows that for a certain distance $d_{i j m}$ between the points $x_{i m}$ and $x_{j m}$ the covariance function becomes zero, meaning that the values of the output at these locations are not correlated. This allows the cubic covariance function to capture variations on smaller length scales and include outliers in the model.

The regression process now consists of selecting the parameters $\left\{\theta_{m}, \sigma^{2}, \sigma_{v}^{2}\right\}$. This is done by maximizing the following equation:

$$
\mathscr{M}=\log p\left(\mathbf{t}_{N} \mid \mathbf{X}_{N},\left\{\theta_{m}, \sigma^{2}, \sigma_{v}^{2}\right\}\right)=-\frac{1}{2} \mathbf{t}_{N}{ }^{T} \mathbf{C}^{-1} \mathbf{t}_{N}-\frac{1}{2} \log |\mathbf{C}|-\frac{n}{2} \log 2 \pi
$$

It should be noted that Gaussian processes are not designed to actually find a specific function $y(\mathbf{x})$ that fits the data best. They are designed to make predictions of the outcome for a new input point $\mathbf{x}_{*}$, without specifying the actual function $y(\mathbf{x})$ that was used to reach that conclusion. For every input $\mathbf{x}_{*}$, the result of the Gaussian process will be a mean $\bar{t}_{*}$ and a variance $\sigma_{\bar{t}_{*}}^{2}$ on the prediction. Using

$$
\mathbf{C}_{*}=\left[C\left(x_{*}, x_{1}\right) C\left(x_{*}, x_{2}\right) \cdots C\left(x_{*}, x_{N}\right)\right] \quad \text { and } \quad \mathbf{C}_{* *}=C\left(x_{*}, x_{*}\right),
$$

the mean and variance of $t_{*}$ become [3]:

$$
\begin{aligned}
P\left(t_{*} \mid \mathbf{t}_{N}\right) & \sim \mathscr{N}\left(\bar{t}_{*}, \sigma_{t_{*}}^{2}\right) \\
& \sim \mathscr{N}\left(\mathbf{C}_{*} \mathbf{C}^{-1} \mathbf{t}_{N}, \mathbf{C}_{* *}-\mathbf{C}_{*} \mathbf{C}^{-1} \mathbf{C}_{*}^{T}\right) .
\end{aligned}
$$

The result of a Gaussian process regression are usually visualised by plotting the mean $\bar{t}_{*}$ as a function of the different inputs $x_{m}$ and adding a confidence interval to the graph, calculated by using the variance $\sigma_{t_{*}}^{2}$ (figure 4 ).

\subsection{Interpretation of the results}

After statistically processing the simulation outcomes with ANOVA or Gaussian processes, the most influential parameters and possible interactions between parameters can be determined. Moreover, a biological interpretation of the predictions might lead to a greater understanding of the modelled processes at hand. Remark, however, that the results of the sensitivity analysis are valid within the chosen parameter as well as response space. Indeed, if the parameter ranges or the responses would be altered, different results could be obtained. Moreover, DOE is a statistical 


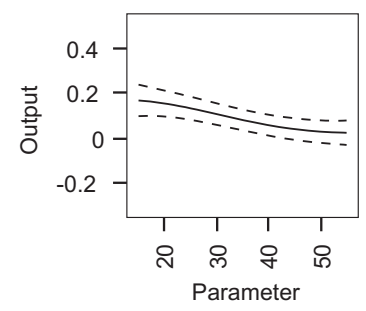

Fig. 4: Visualisation of the results of a Gaussian process. The horizontal axis shows the parameter value, the vertical axis shows the output value predicted by the Gaussian process. For every parameter value, the predicted output has a mean (solid line) and a confidence interval (dashed line).

tool implying that a larger number of runs will provide the statistical model with more data thereby allowing more accurate predictions. It is instructive to test the statistical predictions by running the original model with the according parameter values in order to test whether the examined region was correctly sampled. Finally, every design and analysis method has its pros and cons. If little is known about possible non-linearities, interactions and monotonicity of the model being studied, it might be informative to compare the results of different designs and analysis methods.

\section{Application to a mechanobiological model of tooth movement}

This section demonstrates the techniques described previously by applying them to a mechanobiological model describing tooth movement. Progress in medicine and higher expectation of quality of life have led to a higher demand for several dental and medical treatments [30], making it more and more common for other medical conditions needing to be taken into account by the orthodontist when planning orthodontic treatment. During treatment, tooth displacement is achieved by applying orthodontic forces to the tooth. Under the influence of these forces, the pressure side of the tooth root will experience bone resorption while bone formation will take place on the tension side. The coordination of these two processes through cellular communication results in permanent tooth displacement through the alveolar bone. Together with experiments, computer models might lead to a better understanding of orthodontic treatment and the pathologies affecting the outcome. The model analysed by DOE in this section is a mechanobiological model using partial differential equations to describe cell densities, growth factor concentrations and matrix densities occurring during orthodontic tooth movement and was presented previously by the authors [34]. 


\subsection{Model equations}

The mechanobiological model consists of a set of nine coupled non-linear partial differential equations, of the taxis-diffusion-reaction (TDR) type. The equations describe the concentration of various cells, growth factors, cytokines and matrixcomponents. The periodontal ligament (PDL) consists of collagen fibres $\left(m_{f}\right)$ and contains a large amount of fibroblasts $\left(c_{f}\right)$. The alveolar bone consists of mineralized collagen, with $m_{m}$ representing the degree of mineralization of the collagen. The bone has a small concentration of osteoblasts $\left(c_{b}\right)$ and osteoclasts $\left(c_{l}\right)$, constantly remodelling and renewing the bone. To coordinate bone remodelling, osteoclasts, osteoblasts and fibroblasts communicate through the RANKL-RANK-OPG signalling pathway. In the model, RANKL $\left(g_{r}=g_{r b}+g_{r f}\right)$ is produced by fibroblasts $\left(g_{r f}\right)$ and osteoblasts $\left(g_{r b}\right)$, while OPG $\left(g_{o}\right)$ is produced by osteoblasts only. The osteogenic differentiation of mesenchymal stem cells into osteoblasts is regulated by active TGF- $\beta\left(g_{b}\right)$, also produced by osteoblasts and fibroblasts. Multinucleated osteoclasts are formed through the fusion of hematopoietic stem cells, which are present in the vascular matrix in the PDL and the bone.

Fibroblasts are modelled to respond to mechanical stretching by producing the osteogenic growth factor TGF- $\beta$, along with other osteogenic factors of the TGF- $\beta$ superfamily $[36,14,22,27]$. The upregulation of the TGF- $\beta$ production results in the appearance of a high number of osteoblasts in and around the PDL. This leads to bone formation in the tension zones. Fibroblasts respond to compression by upregulating the production of RANKL $[13,25,37,15]$. This results in a higher number of osteoclasts, which start resorbing the alveolar bone, making it possible for the tooth to move. A schematic overview of the processes captured by the model can be found in figure 5. More information concerning the biological assumptions made in this model can be found in Van Schepdael et al. [34, 35] and a more comprehensive overview of the biology of tooth movement can be found in Garant [7], Krishnan and Davidovitch [16, 15] and Henneman et al. [10].

The specific equations for all nine variables are represented below. More information on the parameters, equations and initial conditions can be found in Van Schepdael et al. [34]. An overview of the origin and value of all parameters can be found in Table 1 .

$$
\begin{aligned}
\frac{\partial m_{c}}{\partial t} & =\underbrace{P_{c s}\left[1-\kappa_{c} m_{c}\right] c_{b}}_{\text {production by osteoblasts }}+\underbrace{P_{c s f}\left[1-\kappa_{c f} m_{c}\right] c_{f}}_{\text {production by fibroblasts }} \\
\frac{\partial m_{m}}{\partial t} & =\underbrace{P_{m s}\left[1-m_{m}\right] c_{b}}_{\text {mineralisation by osteoblasts }}-\underbrace{Q_{m d} c_{l} H\left(m_{m}\right)}_{\text {demineralisation by osteoclasts }} \\
\frac{\partial c_{b}}{\partial t} & =\underbrace{\frac{Y_{11} g_{b}}{H_{11}+g_{b}}\left[1-m_{m}\right] H\left(\bar{m}_{m}-m_{b t}\right)}_{\text {differentiation from MSCs }}+\underbrace{A_{b 0} m_{m} c_{b}\left[1-\alpha_{b} c_{b}\right]}_{\text {proliferation }}-\underbrace{d_{b} c_{b}}_{\text {apoptosis }}
\end{aligned}
$$




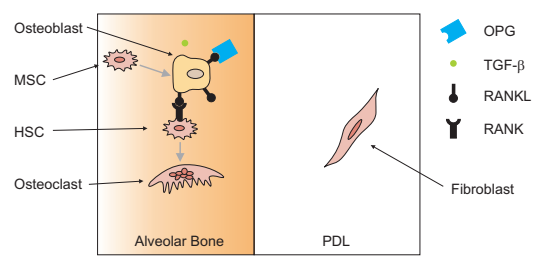

(a)

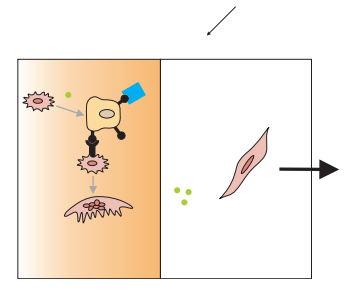

(b)

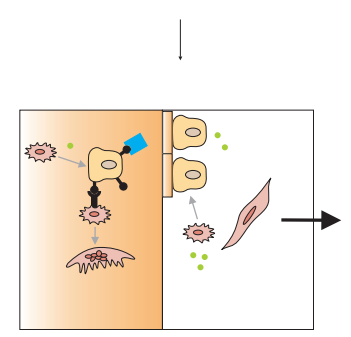

(c)

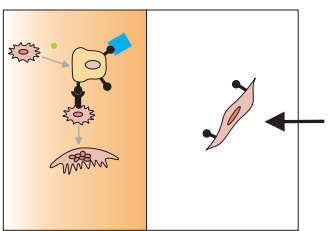

(d)

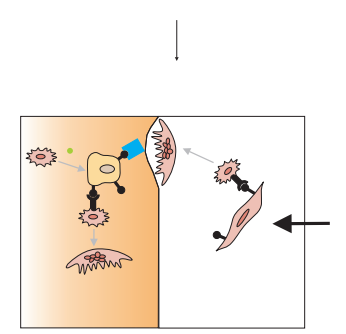

(e)

Fig. 5: Schematic overview of the most important cells and signalling pathways involved in orthodontic tooth movement. a In homeostasis, the fibroblast is the most abundant cell in the PDL, while osteoclasts and osteoblasts are present in the alveolar bone. Osteoblasts are derived from MSC's, which differentiate under the influence of TGF- $\beta$. Osteoclasts are derived from HSC's, which express RANK on their membranes. When RANK binds to RANKL, HSC's are stimulated to fuse into multinucleated osteoclasts. OPG is a soluble decoy operator that also binds to RANKL, thus preventing the formation of osteoclasts. $\mathbf{b}$ - $\mathbf{c}$ In response to tension, fibroblasts express TGF- $\beta$. As a response, MSC's differentiate into osteoblasts, which start forming new bone. $\mathbf{d}$ - e In response to compression, fibroblasts express RANKL, stimulating osteoclast formation. Osteoclasts attach to the bone surface and bone resorption starts. 


$$
\begin{aligned}
& \frac{\partial c_{l}}{\partial t}=\underbrace{Y_{2} g_{r}}_{\text {fusion from HSCs }}-\underbrace{\left[D_{2}+H_{2} g_{b}\right] d_{l 0} c_{l}}_{\text {apoptosis }}-\underbrace{\nabla \cdot\left[C_{m h} c_{l} \nabla m_{m}\right]}_{\text {attachment to bone matrix }} \\
& \frac{\partial c_{f}}{\partial t}=\underbrace{A_{f 0}\left[1+A_{f s}|S|\right] m_{c} c_{f}\left[1-\alpha_{f} c_{f}\right]}_{\text {proliferation }}-\underbrace{d_{f} m_{m} c_{f}}_{\text {apoptosis }}+\underbrace{\Phi \nabla \cdot\left[D_{f} \nabla c_{f}\right]}_{\text {diffusion }} \\
& \frac{\partial g_{b}}{\partial t}=\underbrace{G_{g b}\left[1-\alpha_{g} g_{b}\right] c_{b}}_{\text {production by osteoblasts }}+\underbrace{E_{g b}[S \cdot H(S)] c_{f}}_{\text {production by fibroblasts }}-\underbrace{d_{g b} g_{b}}_{\text {denaturation }}+\underbrace{\nabla \cdot\left[D_{g b} \nabla g_{b}\right]}_{\text {binding to OPG }} \\
& \frac{\partial g_{r b}}{\partial t}=\underbrace{P_{r s}\left[1-\frac{g_{r b}}{R_{1} c_{b}}\right]}_{\text {production by fibroblasts }}-\underbrace{d_{g r} g_{r b}}_{\text {denaturation }}-\underbrace{B_{1 r} g_{r b} g_{o}}_{\text {binding to OPG }} \\
& \frac{\partial g_{r f}}{\partial t}=\underbrace{E_{g r f}[[H(S)-1] S] c_{f}}_{\text {production by fibroblasts }}-\underbrace{d_{g r} g_{r f}}_{\text {denaturation }}-\underbrace{B_{1 r} g_{r f} g_{o}}_{\text {binding to OPG }} \\
& \underbrace{+\nabla \cdot\left[\frac{D_{f}}{c_{f}} g_{r f} \nabla c_{f}\right]}_{\text {moving with fibroblasts }}-\underbrace{d_{f} m_{m} g_{r f}}_{\text {apoptosis of fibroblasts }} \\
& \frac{\partial g_{o}}{\partial t}=\underbrace{P_{o s}\left[1-\kappa_{o} g_{o}\right] c_{b}}_{\text {production by osteoblasts }}-\underbrace{d_{g o} g_{o}}_{\text {denaturation }}-\underbrace{B_{1 o} g_{r} g_{o}}_{\text {binding to RANKL }}+\underbrace{\nabla \cdot\left[D_{g o} \nabla g_{o}\right]}_{\text {diffusion }}
\end{aligned}
$$

The numerical simulations were performed on a domain that consists of two rectangular parts that represent small sections of the tooth root as shown in figure 6, and are located about halfway between the tooth crown and the tooth apex.

Table 2 shows the initial values of all variables in the PDL and the bone. To prevent the appearance of numerical instabilities, continuous initial conditions were used to model the boundary between PDL and alveolar bone. To simulate an abrupt, but continuous, transition from PDL to alveolar bone, the following function was used.

$$
c_{i}(t=0)=\frac{c_{i, \text { bone }}^{0}-c_{i, P D L}^{0}}{\pi} \arctan \left(D \cdot\left[x-x_{S}\right]\right)+\frac{c_{i, \text { bone }}^{0}-c_{i, P D L}^{0}}{2}+c_{i, P D L}^{0}
$$

The parameter $D$ is dependent upon the desired steepness of the function, $x_{S}$ is the $x$-coordinate of the boundary between PDL and alveolar bone, and $c_{i, b o n e}^{0}$ and $c_{i, P D L}^{0}$ are the initial conditions of the variable in the alveolar bone and the PDL.

The model parameters and variables were non-dimensionalised for the numerical calculations. A typical length scale during orthodontic tooth movement is the thickness of the periodontal ligament, $L_{0}=0.2 \mathrm{~mm}$ [29] and a typical time scale of $T_{0}=1$ day was chosen. A representative concentration of collagen content in the tissue is $m_{0}=0.1 \mathrm{~g} / \mathrm{ml}$ [9]. Typical growth factor concentrations are in the order of magnitude of $g_{0}=100 \mathrm{ng} / \mathrm{ml}$, and a non-dimensionalisation value of $c_{0}=10^{6}$ cells $/ \mathrm{ml}$ was used for the cell densities [9]. All results and parameter values are presented in their undimensionalised value, unless mentioned otherwise. 
Table 1: Overview of the parameters of the mechanobiological model, their value, unit and origin. (1) Derived from Geris et al. [9]. (2) Derived from Pivonka et al. [28]. (3) Derived from Pfeilschifter et al. [26] (4) Derived from Sandberg et al. [32].

\begin{tabular}{|c|c|c|c|}
\hline Parameter & Value & Unit & Origin \\
\hline$P_{m s}$ & $3.42 \times 10^{-5}$ & mlcells ${ }^{-1}$ day $^{-1}$ & From steady state conditions \\
\hline$Q_{m d}$ & $3.6 \times 10^{-5}$ & mlcells ${ }^{-1}$ day $^{-1}$ & (1) \\
\hline$P_{c s}$ & $2 \times 10^{-7}$ & gcells $^{-1}$ day $^{-1}$ & (1) \\
\hline$\kappa_{c}$ & 13.55 & $m l g^{-1}$ & (1) \\
\hline$P_{c s f}$ & $2 \times 10^{-8}$ & gcells $^{-1}$ day $^{-1}$ & (1) \\
\hline$\kappa_{c f}$ & 10 & $m g^{-1}$ & (1) \\
\hline$A_{b 0}$ & 0.54 & $d a y^{-1}$ & (1) and stability analysis \\
\hline$\alpha_{b}$ & $2 \times 10^{-5}$ & ml cells ${ }^{-1}$ & (1) and stability analysis \\
\hline$d_{b}$ & 0.18 & $d a y^{-1}$ & (1) \\
\hline$Y_{11}$ & $3.27 \times 10^{8}$ & cellsml $^{-1}$ day $^{-1}$ & (1) \\
\hline$H_{11}$ & 10 & $n g m l^{-1}$ & (1) \\
\hline$m_{b t}$ & 0.3 & {$[-]$} & Estimated \\
\hline$C_{m h}$ & $3.06 \times 10^{-2}$ & $m m^{2} d a y^{-1}$ & Estimated \\
\hline$Y_{2}$ & 551.6 & cellsng ${ }^{-1}$ day $^{-1}$ & (2) \\
\hline$d_{l 0}$ & 0.7 & $d a y^{-1}$ & Using life span of osteoclast \\
\hline$D_{f}$ & 0.25 & $m m^{2}$ day $^{-1}$ & (1) \\
\hline$A_{f 0}$ & 1.06 & $m l g^{-1}$ day $^{-1}$ & (1) \\
\hline$A_{f s}$ & 10 & {$\left[\begin{array}{lll}0 & 0\end{array}\right]$} & Estimated \\
\hline$\alpha_{f}$ & $1 \times 10^{-6}$ & ml cells $s^{-1}$ & (1) \\
\hline$d_{f}$ & 0.11 & $d a y^{-1}$ & From steady state conditions \\
\hline$D_{g b}$ & $6.13 \times 10^{-2}$ & $m m^{2}$ day $^{-1}$ & (1) \\
\hline$G_{g b}$ & $6.03 \times 10^{-5}$ & ngcells $^{-1}$ day $^{-1}$ & (1) \\
\hline$\alpha_{g}$ & 0.1 & $m l^{n} g^{-1}$ & $(3),(4)$ \\
\hline$E_{g b}$ & $1 \times 10^{-4}$ & $n_{\text {gcells }}{ }^{-1}$ day $^{-1}$ & Estimated \\
\hline$d_{g} b$ & 100 & $d a y^{-1}$ & (1) \\
\hline$P_{r s}$ & 3440 & $n g m l^{-1} d a y^{-1}$ & (2) \\
\hline$R_{1}$ & $9.15 \times 10^{-5}$ & ng cells $^{-1}$ & (2) \\
\hline$d_{g r}$ & 10.05 & $d a y^{-1}$ & (2) \\
\hline$B_{1 r}$ & $2.5 \times 10^{-3}$ & $m \operatorname{lng} g^{-1} d a y^{-1}$ & (2) \\
\hline$B_{1 o}$ & $1.67 \times 10^{-3}$ & $m \operatorname{lng}^{-1} d_{a y}^{-1}$ & (2) \\
\hline$E_{g r f}$ & $1 \times 10^{-3}$ & $n_{\text {gcells }}{ }^{-1}$ day $^{-1}$ & Estimated \\
\hline$D_{g o}$ & $4.58 \times 10^{-2}$ & $m m^{2} d a y^{-1}$ & Using molecular weight of OPG \\
\hline$P_{O S}$ & $6.83 \times 10^{-3}$ & $n g$ cells $s^{-1}$ day $^{-1}$ & (2) \\
\hline$\kappa_{o}$ & $8.3 \times 10^{-8}$ & $m l^{n} g^{-1}$ & (2) \\
\hline$d_{g o}$ & 35 & day $^{-1}$ & $(2)$ \\
\hline $\mathrm{D}_{2}$ & 248.5 & {$[-]$} & From $\mathrm{H}_{2}$ \\
\hline $\mathrm{H}_{2}$ & 48.6 & $m \ln g^{-1}$ & (2) \\
\hline
\end{tabular}




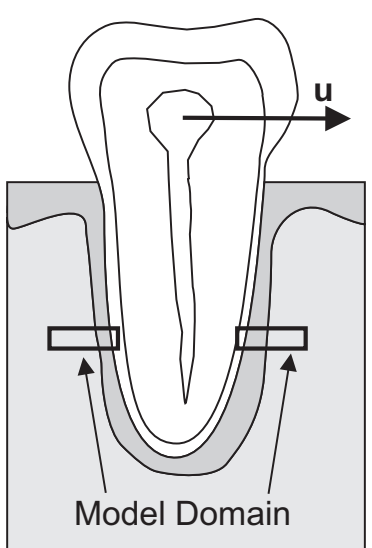

(a)

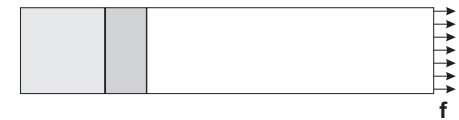

(b)

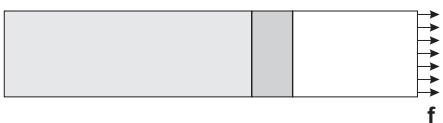

(c)

Fig. 6: Schematic representation of the model domain. (a) The model domain consists of two rectangular parts that are located on the left and the right side of the tooth root. For the simulations, a horizontal translation $\mathbf{u}$ is applied to the root. The left part of the domain will thus experience bone formation, the right part experiences bone resorption. (b) Detail of the formation side of the model domain at the start of force application. (c) Detail of the formation side of the model domain at the end of the simulation. Due to bone formation, more alveolar bone is now present, and the PDL has shifted to the right.

Table 2: Initial conditions applied to the model domain. The initial values and units of all variables in the PDL, the alveolar bone and the root are shown.

\begin{tabular}{lrrrr}
\hline & PDL & Alveolar Bone & Root & Unit \\
\hline$m_{m}$ & 0 & 0.9 & 0 & {$[-]$} \\
$m_{c}$ & 1 & 0.075 & 0 & $\mathrm{~g} \mathrm{ml}^{-1}$ \\
$c_{b}$ & 0 & $3.2 \times 10^{4}$ & 0 & eells ml $^{-1}$ \\
$c_{l}$ & 0 & $2.3 \times 10^{3}$ & 0 & cells ml $^{-1}$ \\
$c_{f}$ & $1 \times 10^{6}$ & 0 & 0 & eells ml $^{-1}$ \\
$g_{b}$ & 0 & $2 \times 10^{-2}$ & 0 & $\mathrm{ng} \mathrm{ml}^{-1}$ \\
$g_{r b}$ & 0 & 2.9 & 0 & $\mathrm{ng} \mathrm{ml}^{-1}$ \\
$g_{r f}$ & 0 & 0 & 0 & $\mathrm{ng} \mathrm{ml}^{-1}$ \\
$g_{o}$ & 0 & 6.2 & 0 & $\mathrm{ng} \mathrm{ml}^{-1}$ \\
\hline
\end{tabular}




\subsection{Model analysis}

The complexity of the biological processes occurring during orthodontic tooth movement results in a high number of parameters in the mechanobiological model. When performing numerical analysis using such models, it is important to study the behaviour of the model, and its parameters, more closely. That way, stability, numerical accuracy and convergence can be ensured (see also Chapter 2 by Kirk et al. elsewhere in this volume).

The model described in the previous section depends upon 37 parameters, although not all of these are independent. To make good predictions, all the parameters have to be determined as accurately as possible, which is not an easy task (see also Chapter 11 by Mannakee et al. elsewhere in this volume). Some of the parameters do not represent physical processes and cannot be measured. Others represent a combination of several processes, making it more difficult to measure them experimentally. Some of the parameter values are based on experimental values, but although some experiments were conducted in vivo, most of the estimations are based on in vitro experimental results.

A sensitivity analysis can be used to determine the importance of the parameters, as well as important interactions between them. Secondly, a sensitivity analysis can also be used to simplify the model by eliminating insignificant model parameters (see also Chapter 12 by Eriksson et al. elsewhere in this volume). Finally, if unexpected behaviour of the model surfaces during the sensitivity analysis, this behaviour can be studied more closely and corrected if necessary.

Since a space-filling design for the full model would be computationally very demanding and very difficult to interpret, a fractional factorial design was performed first to estimate the importance of the parameters. The results were analysed using ANOVA analysis.

The most important parameters were then studied more closely using spacefilling designs. As will be explained in Sect. 3.2.1, some of the parameter combinations will create instabilities in the mechanobiological model or predict physically impossible situations and have to be excluded from the analysis. Furthermore, the tooth model is highly non-linear. Therefore, latin hypercube designs are probably not the best choice for the analysis, while the fact that uniform designs cope well with the adding and removing of parameter combinations makes them a very good choice to examine the tooth model.

Three different uniform designs were performed: one containing the ten most important parameters, one containing the 12 parameters that were most important to the equilibrium conditions in the PDL and the bone, and one containing the 12 parameters that had the highest influence on bone formation and resorption. The results of all uniform designs were analysed using a Gaussian process with a Gaussian and a cubic covariance function. The designs were generated and analysed using JMP (SAS Institute Inc., Cary, North Carolina, USA). 


\subsubsection{Sensitivity analysis using a fractional factorial design}

A two-level fractional factorial design was used to identify the most important parameters of the model. Of the 37 parameters of the model, 28 were studied, as shown in Table 3. All parameters describing matrix densities at which matrix productions stops $\left(\kappa_{c}, \kappa_{c f}\right)$, growth factor concentrations at which growth factor production stops $\left(\alpha_{g}, \kappa_{o}\right)$ or cell concentrations at which cell proliferation is halted $\left(\alpha_{b}, \alpha_{f}\right)$, were excluded from the analysis, since they do not have a specific physiological meaning and influence the model in a very predictable way [12]. The parameter $B_{1 o}$, describing the reaction rate of OPG and RANKL, is related to $B_{1 r}$ and varied accordingly.

The parameters were investigated at two levels, a high and a low level (see Table 3 ), using a resolution IV array with 64 runs. The use of a resolution IV array guarantees that the main effects will not be confounded with two-factor interactions, while limiting the number or runs required for the analysis. For each parameter combination, the model was run three times, once in steady state conditions, once modelling bone formation and once modelling bone resorption. To assess the results obtained from the study, several output variables were studied.

During steady state, the concentrations of nine variables in both the alveolar bone and the PDL were measured. To assess the ability of the model to represent a meaningful situation, a variable Real was introduced, shown by (30). All 18 variables (nine in the alveolar bone, nine in the PDL), were assigned a boolean parameter $\gamma_{i}$ which is zero or one. The value $\gamma_{i}=1$ was given when a predicted concentration approaches the expected concentration (e.g. $m_{m}$ (bone) $\left.=0.7\right), \gamma_{i}=0$ was assigned to a predicted concentration which is not realistic (e.g. $m_{m}($ bone $)=0$ ). Secondly, when one of the steady states has a positive eigenvalue, the boolean parameter $\gamma$ was set to $\gamma=0$, for two stable steady states, $\gamma$ was equal to one.

$$
\text { Real }=\frac{\gamma+\sum_{i=1}^{18} \gamma_{i}}{19}
$$

A typical parameter combination for which the value of Real is small, is one for which the predicted mineralization of the alveolar bone is equal to zero. The same is true for parameter combinations for which one of the steady states is unstable or for which the mineralization of the PDL is very high.

Next, the ability of the model to combine the alveolar bone and the PDL was also studied. This was done by assessing the variable $v_{S S}$ which is the movement of the boundary between bone and PDL. When $v_{S S} \neq 0$, the boundary between bone and ligament will move, even when no orthodontic force is applied. In that case, it is not possible to model co-existence of the alveolar bone and the PDL, which is not a physiological situation.

During bone formation, three variables were recorded: the speed of tooth movement $\left(v_{F}\right)$, the mineralization of the newly formed bone $\left(m_{m n e w}\right)$ and the concentration of TGF- $\beta$ in the PDL $\left(g_{b F}\right)$. During bone resorption, the speed of tooth movement $\left(v_{R}\right)$ and the concentration of RANKL in the PDL $\left(g_{r R}\right)$ were monitored. 
Table 3: Non-dimensionalised parameter ranges used in the different designs of the sensitivity analysis. For the fractional factorial design, the parameters are varied between a low and a high value, as can be seen in the first column. For the other design, several parameters were selected and varied over approximately the same range. Highlighted numbers indicate ranges that have been corrected in order to avoid non-physiological situations. FF: parameter ranges for the fractional factorial design of Sect. 3.2.1. U: parameter ranges for the uniform design of Sect. 3.2.2. USS: parameter ranges for the uniform design on the equilibrium concentrations (Sect. 3.2.3). UM: parameter ranges for the uniform design on bone formation and resorption (Sect. 3.2.4).

\begin{tabular}{|c|c|c|c|c|c|c|c|c|}
\hline \multirow[t]{2}{*}{ Parameter } & \multicolumn{2}{|c|}{ FF } & \multicolumn{2}{|c|}{$\mathbf{U}$} & \multicolumn{2}{|c|}{ USS } & \multicolumn{2}{|c|}{ UM } \\
\hline & Low & High & Low & High & Low & High & Low & High \\
\hline$P_{m s}$ & 15 & 60 & 15 & 60 & & & 15 & 60 \\
\hline$Q_{m d}$ & 18 & 72 & & & 18 & 72 & 18 & 72 \\
\hline$P_{c s}$ & 1 & 4 & & & & & & \\
\hline$P_{c s f}$ & 0.1 & 0.4 & & & & & 0.1 & 0.4 \\
\hline$A_{b 0}$ & 0.25 & 1 & & & & & 0.25 & 1 \\
\hline$d_{b}$ & 0.07 & 0.3 & 0.07 & 0.2 & 0.07 & 0.3 & 0.07 & 0.25 \\
\hline$Y_{11}$ & 180 & 600 & 180 & 600 & 180 & 600 & 180 & 600 \\
\hline$H_{11}$ & 0.05 & 0.2 & 0.05 & 0.2 & 0.05 & 0.2 & & \\
\hline$m_{b t}$ & 0.15 & 0.6 & 0.15 & 0.6 & & & 0.15 & 0.4 \\
\hline$C_{m h}$ & 0.3 & 1.4 & 0.5 & 1.4 & 0.3 & 1.4 & & \\
\hline$Y_{2}$ & 0.02 & 0.1 & & & 0.02 & 0.1 & & \\
\hline$d_{l 0}$ & 0.35 & 1.5 & & & & & & \\
\hline$D_{f}$ & 3 & 9 & & & & & & \\
\hline$A_{f 0}$ & 0.05 & 0.2 & & & 0.05 & 0.2 & & \\
\hline$A_{f s}$ & 5 & 20 & & & 5 & 20 & & \\
\hline$d_{f}$ & 0.05 & 0.2 & & & 0.05 & 0.2 & & \\
\hline$D_{g b}$ & 0.6 & 3 & & & & & & \\
\hline$G_{g b}$ & 0.3 & 1.2 & 0.5 & 1.2 & 0.3 & 1.2 & & \\
\hline$E_{g b}$ & 0.5 & 2 & & & & & 0.5 & 2 \\
\hline$d_{g b}$ & 50 & 200 & 50 & 200 & 50 & 200 & 50 & 150 \\
\hline$P_{r s}$ & 15 & 70 & & & & & & \\
\hline$R_{1}$ & 0.5 & 2 & & & 0.5 & 2 & & \\
\hline$d_{g r}$ & 5 & 20 & 5 & 20 & & & 5 & 20 \\
\hline$B_{1 r}$ & 0.07 & 0.3 & & & & & & \\
\hline$E_{g r f}$ & 5 & 20 & & & & & 5 & 20 \\
\hline$D_{g o}$ & 0.5 & 2 & & & & & & \\
\hline$P_{o s}$ & 40 & 120 & & & & & & \\
\hline$d_{g o}$ & 20 & 70 & & & & & 20 & 70 \\
\hline
\end{tabular}


Steady State

The data were investigated using analysis of variance (ANOVA) and the percentage of the total sum of square, \%TSS, for each parameter and output variable can be found in Table 4 and Table 5. The parameters that most influenced the alveolar bone were mostly related to the concentration of TGF- $\beta$. Both the production rate $G_{g b}$ and the degradation rate $d_{g b}$ have a high influence on many output variables. The same is true for the parameter $H_{11}$, related to the sensitivity of MSC's to TGF- $\beta$. The rate at which osteoclasts demineralize bone $\left(Q_{m d}\right)$ and the haptotactic parameter $C_{m h}$ also seemed to influence the alveolar bone.

Table 4: Results of the fractional factorial design concerning the parameters Real and $v_{S S}$. The percentage of total sum of squares $(\% T S S)$ is listed for each parameter and output variable.

\begin{tabular}{lrrlcr}
\hline & Real & $v_{S S}$ & & Real & $v_{S S}$ \\
\hline$A_{b 0}$ & 8.40 & 0.00 & $E_{g b}$ & 1.27 & 0.00 \\
$A_{f 0}$ & 0.03 & 0.00 & $E_{g r f}$ & 0.03 & 0.71 \\
$A_{f s}$ & 0.00 & 0.71 & $G_{g b}$ & 4.38 & 5.87 \\
$B_{1 r}$ & 0.65 & 0.00 & $H_{11}$ & 0.03 & 5.91 \\
$C_{m h}$ & 3.14 & 0.00 & $m_{b t}$ & 0.03 & 5.87 \\
$d_{b}$ & 24.93 & 0.00 & $P_{c s}$ & 0.93 & 0.00 \\
$D_{f}$ & 1.27 & 0.72 & $P_{c s f}$ & 0.03 & 0.70 \\
$d_{f}$ & 2.59 & 0.00 & $P_{m s}$ & 1.27 & 0.74 \\
$d_{g b}$ & 2.59 & 5.99 & $P_{o s}$ & 1.66 & 0.00 \\
$d_{g o}$ & 0.93 & 5.91 & $P_{r s}$ & 0.00 & 0.00 \\
$d_{g r}$ & 0.23 & 5.91 & $Q_{m d}$ & 4.38 & 0.00 \\
$D_{g b}$ & 1.66 & 0.00 & $R_{1}$ & 0.93 & 5.95 \\
$D_{g o}$ & 0.10 & 0.70 & $Y_{2}$ & 3.14 & 0.00 \\
$d_{l 0}$ & 0.10 & 0.00 & $Y_{11}$ & 0.10 & 5.87 \\
\hline
\end{tabular}




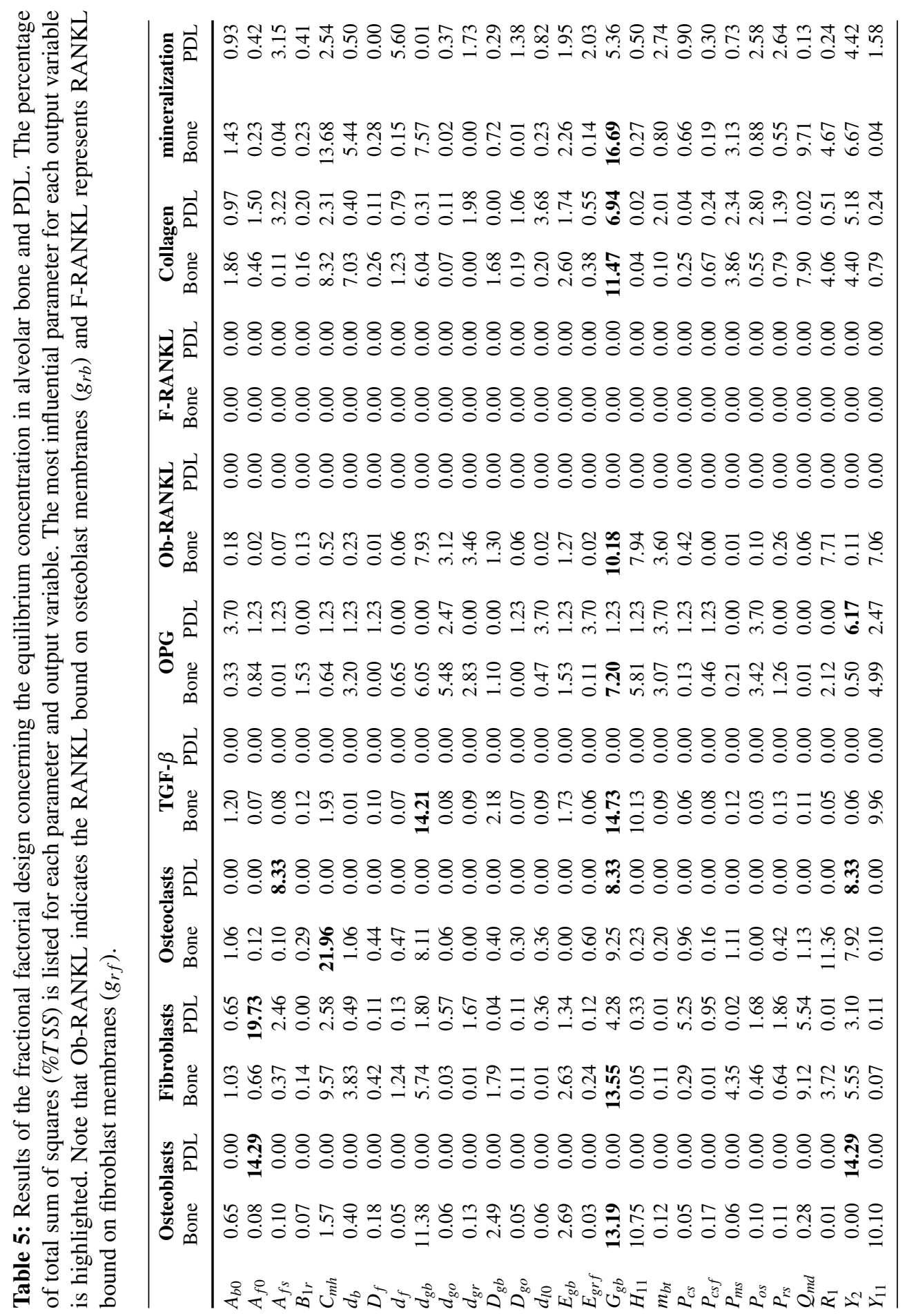


The periodontal ligament was mainly influenced by the proliferation and apoptosis rate of the fibroblasts $\left(A_{f 0}\right.$ and $\left.d_{f}\right)$, the fusion and apoptosis of osteoclasts $\left(Y_{2}\right.$ and $\left.d_{l 0}\right)$ and the production of TGF- $\beta\left(G_{g b}\right)$. The artificial variable Real was most influenced by parameters $A_{b 0}$ and $d_{b}$, describing the proliferation and apoptosis of osteoblasts. The influence of the parameters on $v_{S S}$ was limited.

Out of the 64 parameter combinations chosen for the screening design, 17 gave results where the mineralization $m_{m}$ in the alveolar bone was smaller than 0.1 . In that case, the modelled situation is assumed to be non-physiological, and no results were obtained for bone formation and bone resorption. To prevent this in future analyses, the parameter combinations in which this problem occurred were examined more closely. Without making definite conclusions, the problem seemed to occur more frequently for high values of $Q_{m d}, d_{b}$ and $Y_{2}$ and for low values of $C_{m h}$ and $G_{g b}$. In future analyses care must be taken not to allow parameter combinations that promote bone resorption too strongly, especially when the object is to study bone formation or resorption.

Bone formation and bone resorption

The same process was applied to analyse the data concerning bone formation and bone resorption. The percentage of the total sum of squares of the relevant output variables can be found in Table 6. As expected, the speed of bone formation was mostly influenced by the parameter $m_{b t}$, determining when a mesenchymal stem cell is close enough to the bone to differentiate into an osteoblast. The mineralization rate of collagen by osteoblasts also influenced the speed of bone formation. The concentration of TGF- $\beta$ in the PDL during tooth movement was mainly influenced by the production of TGF- $\beta$ by fibroblasts $\left(E_{g b}\right)$ and the degradation rate $d_{g b}$. This degradation rate also influenced the mineralization of the newly formed bone, just like the parameter $m_{b t}$ and the proliferation rate $A_{b 0}$ of the alveolar bone.

The speed of bone resorption was mainly influenced by the degradation rate $d_{b}$ of osteoblasts and the denaturation rate $d_{g r}$ of RANKL, but many other parameters also had an influence on bone resorption. The amount of RANKL in the PDL was mainly influenced by the denaturation rate $d_{g r}$ of RANKL and the production rate of RANKL by fibroblasts $\left(E_{g r f}\right)$.

\section{Preliminary conclusions}

Although the ANOVA process is able to identify the most important parameters in the process, it gives no insight into the effect of these parameters on the outcome. Furthermore, although some of the results are very straightforward, others need some more explaining. The effect of TGF- $\beta$ on alveolar bone and fibroblasts on the PDL is quite clear, but the influence of the parameter $C_{m h}$ on the alveolar bone is more puzzling. In the following sections, the results of the fractional factorial design will be used to set up several uniform designs, analysed using Gaussian 
Table 6: Results of the fractional factorial design concerning bone formation and bone resorption during orthodontic tooth movement. The percentage of total sum of squares $(\% T S S)$ is listed for each parameter and output variable. The two most influential parameters for each output variable are highlighted.

\begin{tabular}{|c|c|c|c|c|c|}
\hline & \multicolumn{3}{|c|}{ Formation } & \multicolumn{2}{|c|}{ Resorption } \\
\hline & $v_{F}$ & $g_{b F}$ & $m_{\text {mnew }}$ & $v_{R}$ & $g_{r R}$ \\
\hline$A_{b 0}$ & 0.00 & 1.03 & 7.77 & 4.51 & 3.96 \\
\hline$A_{f 0}$ & 0.01 & 0.05 & 0.25 & 0.70 & 0.03 \\
\hline$A_{f s}$ & 0.33 & 0.53 & 0.45 & 0.42 & 3.11 \\
\hline$B_{1 r}$ & 0.00 & 0.09 & 1.51 & 0.56 & 0.05 \\
\hline$C_{m h}$ & 0.43 & 0.12 & 0.47 & 0.46 & 1.87 \\
\hline$d_{b}$ & 0.06 & 0.02 & 4.30 & 10.08 & 3.20 \\
\hline$D_{f}$ & 0.66 & 0.13 & 0.08 & 0.07 & 0.21 \\
\hline$d_{f}$ & 0.73 & 0.29 & 1.15 & 3.47 & 0.10 \\
\hline$d_{g b}$ & 0.58 & 37.78 & 9.93 & 7.04 & 0.05 \\
\hline$d_{g o}$ & 0.55 & 1.44 & 0.33 & 0.79 & 5.85 \\
\hline$d_{g r}$ & 0.16 & 0.06 & 0.38 & 10.07 & 28.37 \\
\hline$D_{g b}$ & 0.11 & 0.30 & 0.11 & 0.13 & 0.45 \\
\hline$D_{g o}$ & 1.10 & 0.16 & 4.21 & 1.42 & 0.72 \\
\hline$d_{l 0}$ & 0.10 & 0.03 & 0.15 & 0.79 & 0.08 \\
\hline$E_{g b}$ & 0.10 & 42.07 & 0.02 & 0.56 & 0.01 \\
\hline$E_{g r f}$ & 2.17 & 0.05 & 0.38 & 5.94 & 23.76 \\
\hline$G_{g b}$ & 0.51 & 0.20 & 1.35 & 3.57 & 0.20 \\
\hline$H_{11}$ & 0.41 & 0.63 & 0.64 & 2.97 & 0.80 \\
\hline$m_{b t}$ & 63.24 & 0.23 & 36.30 & 1.38 & 3.92 \\
\hline$P_{c s}$ & 0.00 & 0.15 & 0.27 & 0.04 & 0.01 \\
\hline$P_{c s f}$ & 0.48 & 0.17 & 0.04 & 5.60 & 1.11 \\
\hline$P_{m s}$ & 8.14 & 0.00 & 4.14 & 1.85 & 2.01 \\
\hline$P_{o s}$ & 0.20 & 0.02 & 1.29 & 0.10 & 1.80 \\
\hline$P_{r s}$ & 0.09 & 0.10 & 0.71 & 3.33 & 0.99 \\
\hline$Q_{m d}$ & 0.68 & 0.33 & 6.41 & 3.17 & 0.13 \\
\hline$R_{1}$ & 0.33 & 0.43 & 1.87 & 0.02 & 0.22 \\
\hline$Y_{2}$ & 0.67 & 0.07 & 1.42 & 1.13 & 0.01 \\
\hline$Y_{11}$ & 1.08 & 2.30 & 1.84 & 5.66 & 0.01 \\
\hline
\end{tabular}

processes, that study the influence of these parameters more closely. That way, the exact effect of the parameters can be investigated, and the interaction between parameters can be studied.

\subsubsection{Sensitivity analysis using a uniform design}

To solve the questions that arose in the previous section, it was decided to look more closely at the ten most influential parameters. Those parameters were chosen by selecting every parameter for which $\% T S S>10 \%$. As the high influence of the artificial parameter $m_{b t}$ on the speed of bone formation dominated the results, the parameter $P_{m s}$ was added to this list. From this list, those parameters who influenced 
several output variables or output variables with a large variation, represented by a high value of $S S_{T}$, were chosen. The parameter ranges of $d_{b}, G_{g b}$ and $C_{m h}$ were limited to avoid non-physiological situations in which the mineralization of the alveolar bone was too low (Table 3).

The influence of the ten parameters was investigated as previously explained using a uniform design with 100 runs. 100 parameter combinations were generated, evenly spread out over the ten-dimensional parameter space. The uniformity of the distribution can be checked using scatterplots and histograms (figure 7).

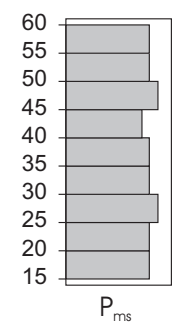

(a)

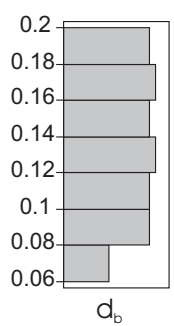

a)

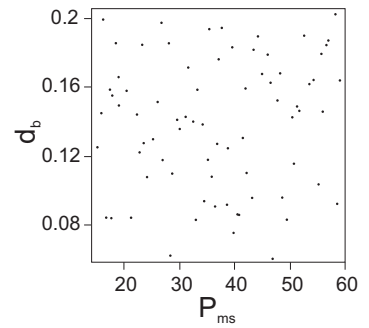

(b)

Fig. 7: Uniformity of the distribution in a uniform design. a Histogram showing the distribution of the parameters $P_{m s}$ and $d_{b}$ in a uniform design. In this particular example, it can be seen that the distribution of $P_{m s}$ is uniform, while for the parameter $d_{b}$, only a small number of low values are included. b Scatterplot of all parameter combinations. The parameter combinations are spread out over the entire parameter space.

The results of a uniform process can no longer be analysed using ANOVA. Since the parameter points are now spread out over the parameters space, the exact value of the parameters has to be taken into account. A Gaussian process with a Gaussian covariance function was therefore used. Afterwards, the results were compared to an analysis using a Gaussian process with a cubic covariance function.

One remark concerning the results of the Gaussian process is that one should take care when interpreting the predictions. When assessing the accuracy of the predictions, two methods can be used. Firstly, looking at the value of the function $\mathscr{M}$, defined in (17), will give a good idea of the efficiency of the process. Secondly, JMP plots the actual results, computed by your model, and compares these to the results predicted by the Gaussian process. Figure 8 illustrates this for the specific examples of mineralization of the alveolar bone and the concentration of fibroblasts in the PDL. As can be seen from this figure, the results for the mineralization of the bone are predicted far more accurate than the results for the concentration of fibroblasts. Since the horizontal axis plots the predicted value and the vertical axis the actual value, all the points would be located on a 45-degree line in a perfect model. A deviation from this line can have various causes. Firstly, because a more sophisticated covariance function may be needed, but secondly, as is the case here, 
because the variation in the output variable is so small the numerical errors become too important. When interpreting the analysis, care should be taken only to include those results with accurate predictions.

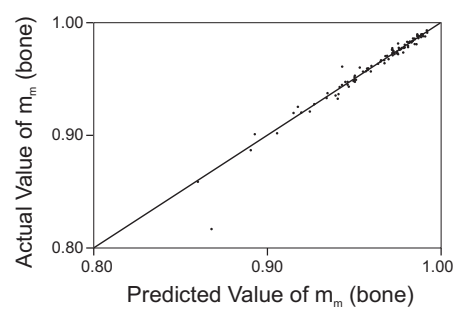

(a)

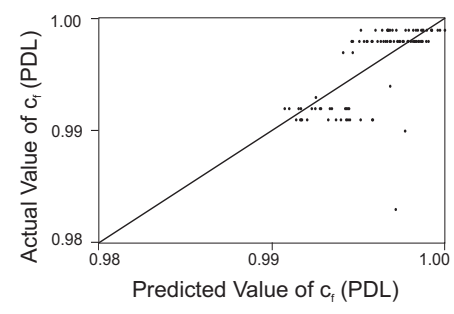

(b)

Fig. 8: Actual by predicted plot for the mineralization of the alveolar bone and the fibroblast density in the PDL. The horizontal axis shows the value of the output predicted by the Gaussian process, the vertical axis shows the actual output value of the mechanobiological model. a Actual by predicted plot for the mineralization of the alveolar bone. b Actual by predicted plot for the fibroblast density in the PDL.

\section{Steady State}

The predictions for the most important output variables are summarized in figure 9 . From these results it can be seen that the mineralization rate $P_{m s}$ of the collagen fibres by osteoblasts positively influences the mineralization of both the alveolar bone and the PDL, and has a negative influence on the amount of fibroblasts in the bone. Increasing the apoptosis rate of osteoblasts $d_{b}$ decreases the number of osteoblasts and the concentrations of RANKL and OPG, both produced by osteoblasts. Raising the production rate $G_{g b}$ of TGF- $\beta$ by osteoblasts increases the mineralization of the bone and the TGF- $\beta$ concentration while decreasing the number of osteoclasts. Increasing the denaturation rate $d_{g b}$ has the exact opposite effect. From this uniform design, it is now clear that increasing $C_{m h}$ seems to favour bone formation by decreasing the number of osteoclasts.

The variable $v_{S S}$ only becomes non-zero for low values of $d_{g b}$, but this result should be taken lightly, since out of 100 parameter combinations, the parameter was non-zero for only two parameter combinations. The results also show that limiting the parameter ranges of $d_{b}, G_{g b}$ and $C_{m h}$ had its desired effect, keeping the mineralization in reasonable bounds. As a result, the output Real has a value of one over the entire parameter space (results not shown). 


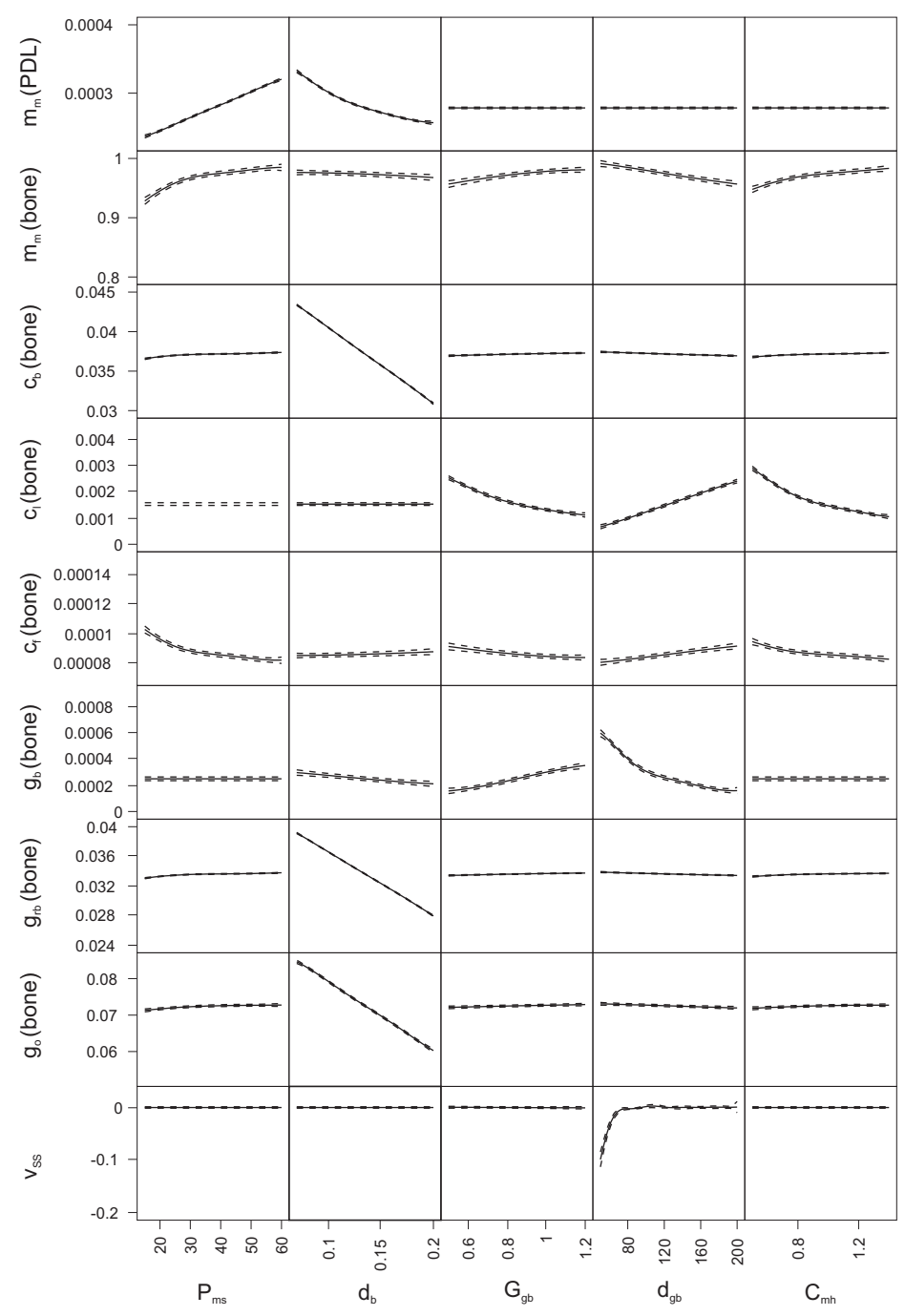

Fig. 9: Results of the uniform design concerning the equilibrium conditions in the alveolar bone and the PDL, and the parameters Real and $v_{S S}$. Only significant results are shown. Solid lines represent the prediction of the non-dimensionalised output variable by the Gaussian process, dashed lines represent the confidence intervals. The first column of figures shows the influence of $P_{m s}$ on several output variables, assuming all other parameters have values in the middle of their range (e.g $d_{b}=0.135$, $G_{g b}=0.85$, etc.). The same holds for the other columns showing the influence of $d_{b}, G_{g b}, d_{g b}$ and $C_{m h}$. 
Bone formation and bone resorption

As was already shown in the ANOVA analysis, the main influence on the speed of bone formation was the parameter $m_{b t}$, although increasing the mineralization rate also increased bone formation. As figure 10 shows, a value of $m_{b t}>0.4$ reduced the speed of bone formation to zero. Decreasing the denaturation rate $d_{g b}$ greatly increased the concentration of TGF- $\beta$ in the PDL.

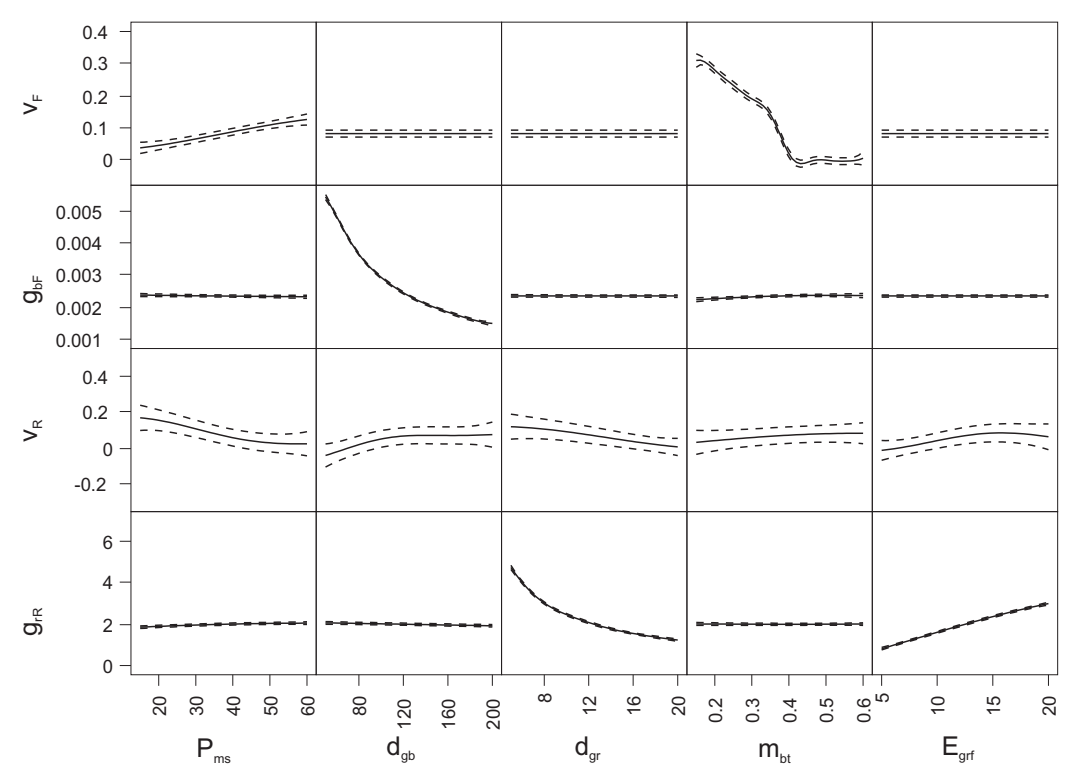

Fig. 10: Results of the uniform design concerning bone formation and resorption during orthodontic tooth movement. Only significant results are shown. Solid lines represent the prediction of the non-dimensionalised output variable by the Gaussian process, dashed lines represent the confidence intervals.

The concentration of RANKL is clearly influenced by the production rate $E_{g r f}$ and the denaturation rate $d_{g r}$, but the speed of bone resorption shows some complex behaviour that will be looked at more closely in the next analysis. In summary, the osteogenic parameters $P_{m s}$ and $G_{g b}$ slow down bone resorption, while increasing $d_{b}$, $E_{g r f}$ or $d_{g b}$ allowed bone to be resorbed faster.

The effect of the choice of the covariance function

In order to study the influence of the covariance function, the analysis was repeated using a cubic covariance function. As discussed in Sect. 2.2.2, the cubic covariance 
function includes smaller length scale, allowing smaller features to be incorporated in the results.

The majority of the results for the cubic covariance function did not differ from the results with the Gaussian covariance function. However, there were three major differences. Firstly, when looking at the results concerning the parameter $d_{b}$ (the apoptosis rate of osteoblasts), $\theta_{m}$ becomes very large $\left(\theta_{m}>50\right)$ for several output variables. Since $1 / \theta_{m}$ represents the length scale of the modelled features, this means that very small variations will be incorporated into the results. As can be seen from figure 11 this does not change the general trend of the results. Since the parameter $d_{b}$ (and many others) represent a physical process, the small variations are most likely due to numerical errors. Although non-linearities occur in nature, changing one parameter will more than likely result in a smooth change of the outcome variable.

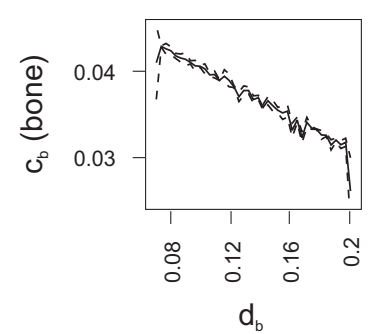

(a)

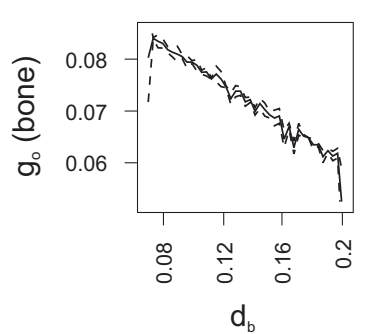

(b)

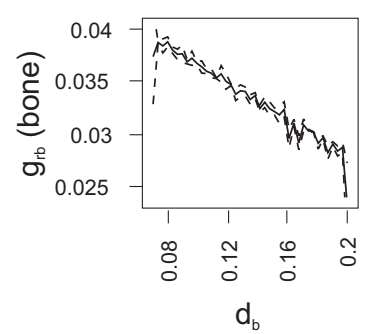

(c)

Fig. 11: Some results of the uniform design obtained using the cubic covariance function. Small fluctuations can be seen in the predicted influence of the osteoblast apoptosis rate $d_{b}$ on the osteoblast density in the alveolar bone, as well as in the influence of $d_{b}$ on the RANKL and OPG concentrations in the alveolar bone.

Secondly, when studying the results for the speed of bone formation and the mineralization of the newly formed bone (figure 12), some highly non-linear behaviour can be seen. Once $m_{b t}$ exceeds $m_{b t}=0.4$, the predicted results for the mineralization of the newly formed bone show a very erratic behaviour. Furthermore, the predicted effect of $H_{11}$ on the speed of bone formation shows similar variations. The cause of this effect can be seen when looking at the marginal model plots for $H_{11}$ and $m_{b t}$, produced by JMP. These are plots that show the actual and the predicted values of an output variable as a function of only one parameter, disregarding the influence of other parameters. Figure 13 shows the actual and predicted values of $v_{F}$ as a function of these two parameters. There is an apparent split into two classes: one for which the speed of bone formation is positive, and one for which it is zero. The latter class appears when $m_{b t}>0.4$, regardless of the values of other parameters. Since the cubic covariance function allows for very small length scales, the Gaussian process attempts to include both classes into one model, when actually they represent very different processes. 


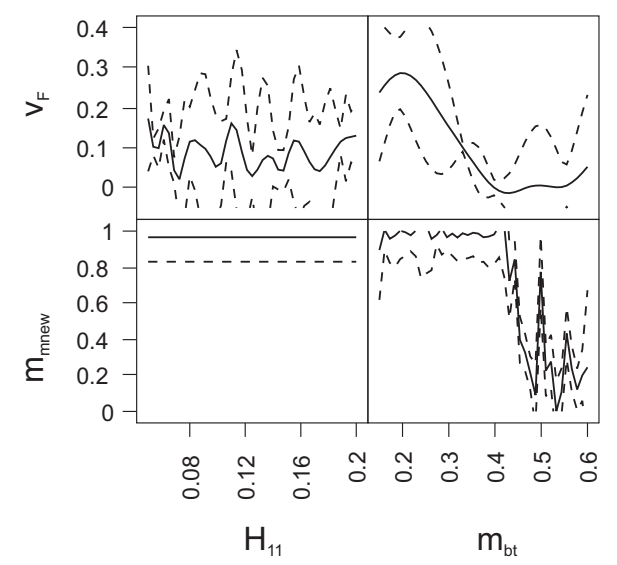

Fig. 12: The influence of $H_{11}$ and $m_{b t}$ on bone formation, using the cubic covariance function, shows some highly non-linear behaviour and very erratic behaviour with very large confidence intervals.

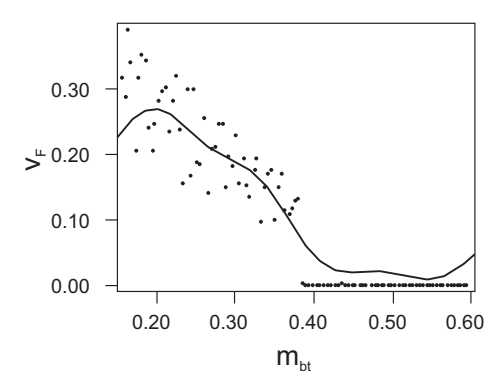

(a)

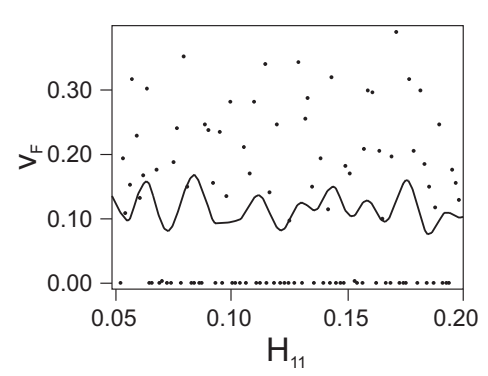

(b)

Fig. 13: Marginal model plots for $H_{11}$ and $m_{b t}$. The horizontal axis shows the value of the parameter, the vertical axis shows the value of the output variable $v_{F}$. The dots indicate actual results obtained using the mechanobiological model, the solid line shows the results predicted by the Gaussian process. a The influence of $m_{b t}$, indicating how close MSC's have to be to the bone to differentiate into osteoblasts, on the speed of bone formation. When $m_{b t}>0.4$, no bone formation occurs. $\mathbf{b}$ The influence of the sensitivity of MSC's to TGF- $\beta$ (determined by $H_{11}$ ) on the speed of bone formation. The actual results can be split up into two classes: one for which there is bone formation, and one for which $v_{F}=0$, located at the bottom. 
Finally, it should be noted that in general, the confidence intervals are much larger when using the cubic covariance function, compared to the Gaussian covariance function. This is particularly apparent in the case of the speed of bone formation. In that case, the plot of the actual value versus the predicted value already indicates a problem when using the cubic covariance function, while for the Gaussian covariance function the results were good (figure 14).

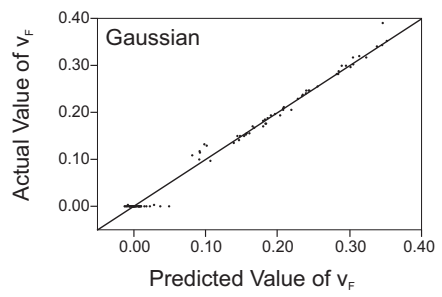

(a)

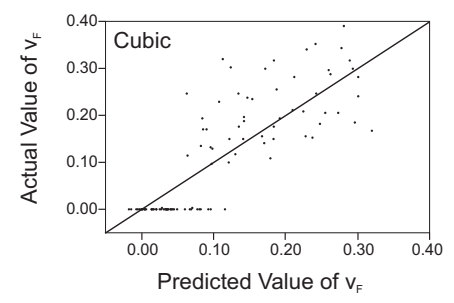

(b)

Fig. 14: Actual by predicted plot for the speed of bone formation, using the Gaussian and the cubic covariance function. a Actual by predicted plot using the Gaussian covariance function. All points are located quite close to the 45-degree line, indicating a good prediction. b Actual by predicted plot using the cubic covariance function. In this case, the points are spread out, indicating the predictions made by the sensitivity analysis for the speed of bone formation using the cubic covariance function are less reliable.

\section{Preliminary conclusions}

From this uniform design, some preliminary conclusions can be drawn. Firstly, the use of the cubic covariance function added little to the value of the analysis. On the contrary, it introduced some errors and problems that did not appear when using the Gaussian covariance function. Secondly, when setting up more designs, care should be taken with the parameter range of $m_{b t}$. To avoid that the speed of bone formation drops to zero, $m_{b t}$ should be kept smaller than 0.4 .

This uniform design was implemented using the ten parameters which proved to be most influential according to the ANOVA analysis of the fractional factorial design explained in Sect. 3.2.1. However, the high number of output variables complicated the analysis of the results. Furthermore, most of the output variables were only influenced by one, or sometimes two, of the parameters. This implies that the uniform design will not give any information on the interaction between different parameters. For these reasons, it was decided to run two more uniform designs. The first one studies the influence of 12 parameters on the steady states, the second one 
looks more closely into the influence of 12 (different) parameters on bone formation and bone resorption.

\subsubsection{Sensitivity analysis on the equilibrium concentrations using a uniform design}

A 12-parameter uniform design with 100 runs was set up. Using the fractional factorial design described in Sect. 3.2.1, 12 parameters were chosen that influenced the steady states the most. In Table 4, the most important parameter was chosen out of each column related to the steady state. This set of seven parameters was then expanded with five parameters which were not included yet, but had a significant influence on one or more outcome variables. Since no results for bone formation or resorption will be calculated, and in order to study the influence of the parameters over the entire parameter space, the parameter ranges were kept the same as in the fractional factorial design.

\section{Steady State}

The parameters can be classified into two sets: those favouring bone formation and those favouring bone resorption. The most significant results of the analysis are shown in figure 15. Increasing the production rate of TGF- $\beta\left(G_{g b}\right)$ increases the mineralization of the bone, while decreasing the osteoclast concentration and the number of fibroblasts in the bone. The apoptosis rate of osteoblasts $\left(d_{b}\right)$, the decay rate of TGF- $\beta\left(d_{g b}\right)$, the demineralization rate of the bone by osteoclasts $\left(Q_{m d}\right)$, the sensitivity of HSC's to RANKL $\left(Y_{2}\right)$ and the maximum RANKL carrying capacity of osteoblasts $\left(R_{1}\right)$ have the opposite effect. They decrease mineralization and osteoblast concentration, while increasing the number of osteoclasts, resulting in a lower mineralization of the bone. As in the previous uniform design, the haptotactic parameter $C_{m h}$ seemed to encourage bone formation by decreasing the number of osteoclasts. Finally, increasing the proliferation rate of fibroblasts $A_{f 0}$ increased the density of the PDL and the concentration of fibroblasts, although the influence was very small and confidence intervals quite large.

The analysis also shows a significant interaction between the parameters $H_{11}$, $G_{g b}$ and $d_{g b}$ when modelling the concentrations of osteoblasts and TGF- $\beta$. When looking at the results of the analysis more closely, it can be concluded that for low values of $H_{11}$ and $d_{g b}$ (figure 16), the model is more non-linear. The influence of $G_{g b}$ becomes more irregular and highly non-linear. The marginal model plots (figure 17) suggest this might be related to the presence of two outliers, in which the concentrations of osteoblasts and TGF- $\beta$ are much higher then average.

These outliers are located in an area of the parameter space with a high sensitivity of MSC's to TGF- $\beta$, described by a low value of $H_{11}$, combined with a long half-life of TGF- $\beta$, expressed by the low value of $d_{g b}$ and an average to high production of TGF- $\beta$ by osteoblasts $\left(G_{g b}\right)$. This particular combination results in a chain reaction 


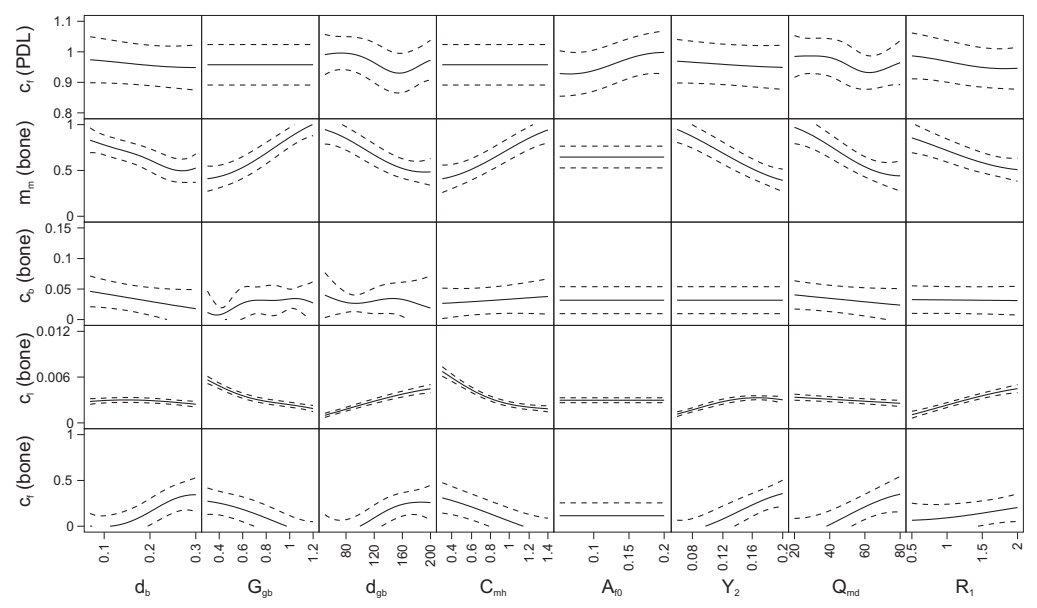

Fig. 15: Results of the uniform design on the equilibrium concentrations. Only significant results are shown. Solid lines represent the prediction of the nondimensionalised output variable by the Gaussian process, dashed lines represent the confidence intervals.

in which the concentration of osteoblasts will rise quickly, producing even more TGF- $\beta$. The fact that the analysis predicts that the concentrations of osteoblasts will return back to normal for high values of $G_{g b}$ can be explained by the lack of parameter combinations in that particular area of the parameter space. This was confirmed by running the model with parameter combinations in that area of the parameter space.

For the variable $v_{S S}$ no conclusions can be drawn, since, out of 100 runs, only three values are non-zero. To prevent non-physiological situations in the next design, the results for the variable Real were interpreted. It was concluded that the appearance of such a situation was most likely with high values of $d_{b}, d_{g b}, Y_{2}, Q_{m d}$ and $R_{1}$, all parameters favouring bone resorption, or with low values of $G_{g b}$ and $C_{m h}$.

The effect of the choice of the covariance function

The results of the uniform design were also analysed using a cubic covariance function. As before, results were generally very similar to the Gaussian covariance analysis. One observed difference is the effect of the parameter $d_{g b}$ on the osteoblast concentration and the presence of TGF- $\beta$, OPG and RANKL in the bone. Figure 18 shows the results obtained using the cubic covariance function, revealing a sharp rise in osteoblast concentrations for small values of $d_{g b}$. Using the Gaussian covariance function, this rise is only seen to that extent when a low value of $d_{g b}$ is combined with a low value of $H_{11}$. The analysis with the cubic covariance function predicts 


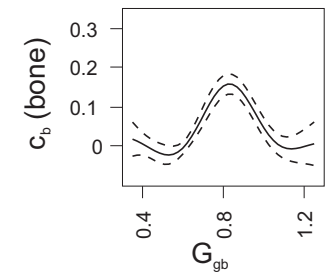

(a)

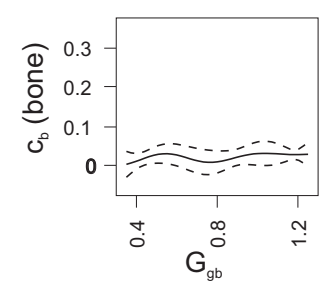

(c)

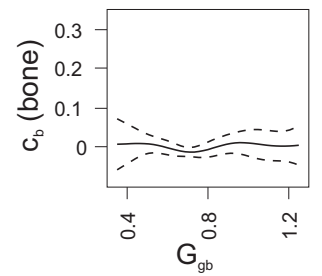

(b)

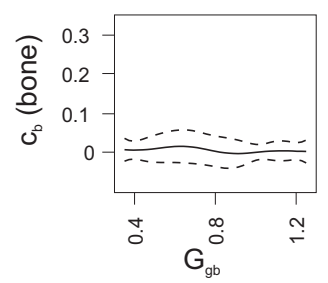

(d)

Fig. 16: Influence of the production rate of TGF- $\beta\left(G_{g b}\right)$ on the predicted osteoblast density in bone, for different values of $H_{11}$ and $d_{g b}$. A low value of $H_{11}$ indicates a high sensitivity of MSC's to TGF- $\beta$ and a low value of $d_{g b}$ coincides with a long half life of TGF- $\beta$. a Results for $H_{11}=0.08$ and $d_{g b}=60$, for which the influence of $G_{g b}$ on the osteoblast density is highly non-linear. b Results for $H_{11}=0.18$ and $d_{g b}=180$, showing a limited influence of $G_{g b}$ on the osteoblast density. c Results for $H_{11}=0.08$ and $d_{g b}=180$, showing a limited influence of $G_{g b}$ on the osteoblast density. d Results for $H_{11}=0.18$ and $d_{g b}=60$, showing a limited influence of $G_{g b}$ on the osteoblast density.

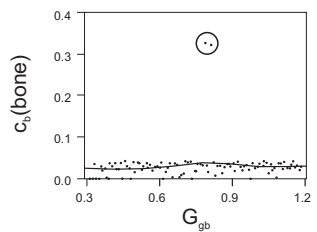

(a)

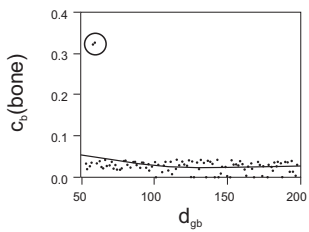

(b)

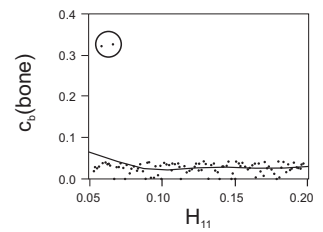

(c)

Fig. 17: Marginal model plots for $G_{g b}, d_{g b}$ and $H_{11}$. The horizontal axis shows the value of the parameter, the vertical axis shows the osteoblast concentration in alveolar bone. The dots indicate actual results obtained using the mechanobiological model, the solid line shows the results predicted by the Gaussian process. On each figure, two outliers (circled) with a very high osteoblast density can be noted. a The influence of the production rate of TGF- $\beta\left(G_{g b}\right)$ on osteoblast density. b The influence of the denaturation rate of TGF- $\beta\left(d_{g b}\right)$ on osteoblast density. $\mathbf{c}$ The influence of the sensitivity of MSC's to TGF- $\beta$ (determined by $H_{11}$ ) on osteoblast density. 
that the chain reaction leading to an alveolar bone with a high osteoblast and TGF$\beta$ content occurs at low values of $d_{g b}$, independent of the sensitivity of MSC's to TGF- $\beta$, described by $H_{11}$. To get a more detailed description of this phenomenon, a more elaborate sensitivity analysis with more points in that region of parameter space is needed.

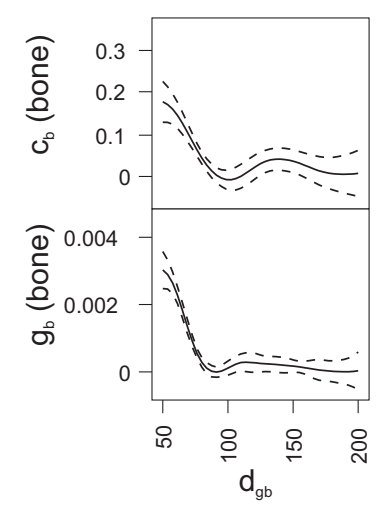

Fig. 18: The influence of $d_{g b}$ on the osteoblast density and TGF- $\beta$ concentration in the alveolar bone, using the cubic covariance function. When using the cubic covariance function a sharp rise in both osteoblast density and TGF- $\beta$ concentration is predicted for low values of $d_{g b}$. This rise is not predicted when using the Gaussian covariance function (figure 15).

\subsubsection{Sensitivity analysis on bone formation and bone resorption using a uniform design}

A 12-parameter uniform design with 100 runs was used to assess the influence of several parameters on tooth movement. Using the fractional factorial design described in Sect. 3.2.1, 12 parameters were chosen that influenced the processes of bone formation and resorption during tooth movement the most. In Table 4, the most important parameter was chosen out of each column. This set of seven parameters was then expanded with five parameters which were not included yet, but had a significant influence on one or more outcome variables.

To prevent the occurrence of non-physiological situations in which the mineralization of the alveolar bone was too low, the parameter ranges of $d_{g b}$ and $d_{b}$ were limited. The upper limit of $m_{b t}$ was also changed to 0.4 , in order to avoid situations in which the speed of bone formation dropped to zero (see Sect. 3.2.2).

As expected, the speed of bone formation was mainly influenced by the parameter $m_{b t}$ (figure 19), but also increased with increasing mineralization rate $P_{m s}$. The mineralization of the newly formed bone decreases when the decay rate of TGF- $\beta$ 
$\left(d_{g b}\right)$ is higher and the presence of TGF- $\beta$ in the ligament was influenced by the production rate $E_{g b}$ of TGF- $\beta$ by fibroblasts and the parameter $d_{g b}$.

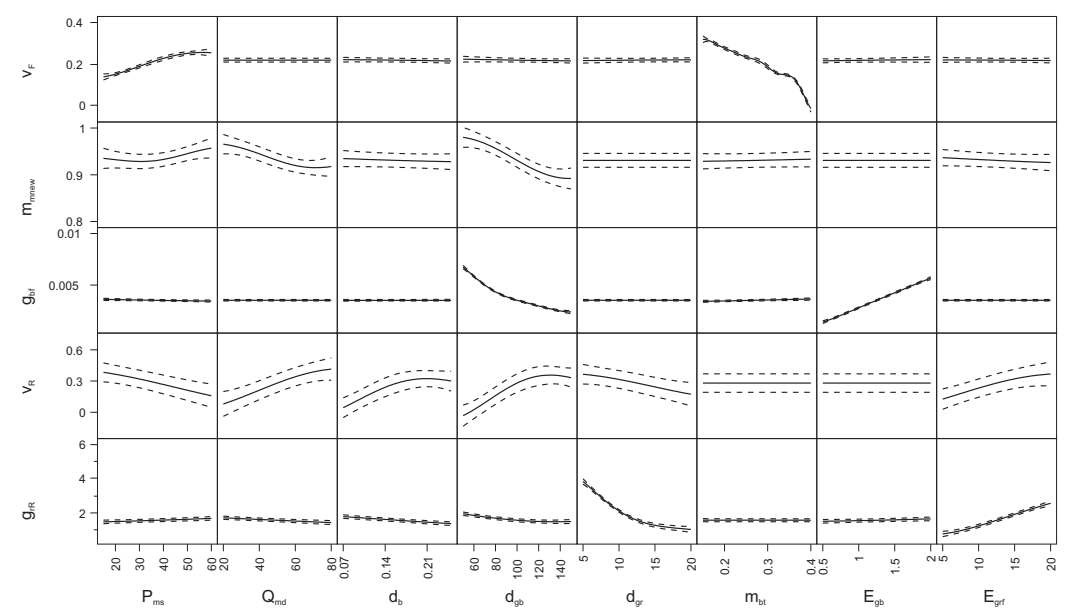

Fig. 19: Results of the uniform design on bone formation and resorption. Only significant results are shown. Solid lines represent the prediction of the nondimensionalised output variable by the Gaussian process, dashed lines represent the confidence intervals.

The effect of the parameters on the resorption rate $v_{R}$ is less clear. Most parameters influence the resorption rate to some extent. Increasing the mineralization rate $P_{m s}$ or the apoptosis rate $d_{g r}$ of RANKL slows down bone resorption during tooth movement. Increasing the demineralization rate $Q_{m d}$, the apoptosis rate $d_{b}$ of osteoblasts and $d_{g b}$ of TGF- $\beta$, or the production $E_{g r f}$ of RANKL by fibroblasts speeds up bone resorption. As expected, the concentration of RANKL in the PDL during bone resorption is determined by the parameters $E_{g r f}$ and $d_{g r}$. Similar results were obtained using the cubic covariance function.

\subsection{Discussion}

The design of experiments was used to determine the most important parameters of the model, and to investigate their result on the outcome of the model. The sensitivity analysis started with a two-level fractional factorial design, followed by an ANOVA analysis of the results. Several parameters where then selected for a more in-depth analysis. Three different uniform designs were performed using these parameters, in order to assess their influence on the alveolar bone, the PDL, and the speed of bone formation and resorption. 
The results of these analyses are summarized in Table 7. During homeostasis, bone resorption was shown to be promoted by the activity of osteoclasts $\left(Q_{m d}\right)$ and their sensitivity to RANKL $\left(Y_{2}\right)$. Increasing the decay constant used to model the apoptosis of osteoblasts $\left(d_{b}\right)$, or the denaturation rate of TGF- $\beta\left(d_{g b}\right)$ had similar effects. The disappearance of both osteoblasts and TGF- $\beta$ from the bone decreased the bone mass. Increasing bone mass was mainly noted as a result of increasing the production of TGF- $\beta\left(G_{g b}\right)$ or the activity of osteoblasts $\left(P_{m s}\right)$.

Table 7: Summary of the parameters that showed the most influence on equilibrium conditions and tooth movement (TM), as indicated by the sensitivity analysis.

\begin{tabular}{ll}
\hline Parameter & Influence \\
\hline$Q_{m d}$ & Promotes bone resorption \\
$d_{b}$ & Promotes bone resorption \\
$Y_{2}$ & Increases bone resorption during TM \\
$G_{g b}$ & Promotes bone resorption \\
$d_{g b}$ & Promotes bone formation \\
& Promotes bone resorption \\
& Increases TGF- $\beta$ concentration during TM \\
$P_{m s}$ & Increases mineralization during TM \\
& Increases bone resorption during TM \\
& Promotes bone formation \\
$m_{b t}$ & Increases bone formation during TM \\
$E_{g b}$ & Decreases bone resorption during TM \\
$d_{g r}$ & Decreases bone formation during TM \\
& Increases TGF- $\beta$ concentration during TM \\
$E_{g r f}$ & Decreases bone resorption during TM \\
& Decreases RANKL concentration during TM \\
\hline & Increases bone resorption during TM \\
\hline
\end{tabular}

Under normal conditions, the model has two separate steady states. The first one represents the PDL, with a high concentration of fibroblasts and no mineralization, the second one represents the alveolar bone, consisting of mineralized collagen fibres, osteoblasts and osteoclasts. Changing the parameters usually resulted in a small, continuous change of the steady states, preserving their main properties. Alveolar bone and PDL remained recognisable as such. However, in some cases more abrupt changes where noted.

Firstly, with several parameter combinations that promoted bone resorption, the predicted mineralization of the alveolar bone dropped to zero. With those parameter combinations, only one steady state was possible and the alveolar bone could not be modelled. Secondly, when a high sensitivity of MSC's to TGF- $\beta$ was combined with a high production and a long half-life of TGF- $\beta$, a chain reaction occurred. The steady state representing the alveolar bone switched from bone containing a small amount of osteoblasts to bone containing large amounts of osteoblasts. Since the parameter combinations at which the phenomenon occurred were located at the edge 
of the parameter space investigated in this analysis, it did not pose any problems. For the same reason, not much information of the phenomenon and its influences on tooth movement was obtained. Performing a sensitivity analysis with a broader parameter space will give a better insight in this chain reaction.

Concerning the parameter $C_{m h}$, describing the haptotaxis of osteoclasts, an unexpected effect was discovered. The modelling of haptotaxis of osteoclasts, attracting them to the alveolar bone, was included in the model to mimic the function of osteopontin and the working of osteoclasts during bone resorption. The fact that increasing $C_{m h}$ seemed to favour bone formation during steady state was unexpected, and initiated a closer investigation of this process. It was concluded that this effect was related to the implementation of the continuous transition between the PDL and the alveolar bone during steady state, as described in (29). Due to this approach, the mineralization in the alveolar bone is not constant, but increases slightly when moving away from the PDL. As a result, with higher values of $C_{m h}$, more osteoclasts will migrate to the more mineralized part of the domain, where osteoclast apoptosis is slightly higher due to the higher concentration of TGF- $\beta$. The number of osteoclasts at the measuring point will thus decrease, resulting in a higher degree of mineralization. It should be noted that, although the effect does occur, it is more subtle than predicted by the sensitivity analysis.

Four parameters had a significant influence on bone formation during orthodontic tooth movement. The activity of osteoblasts $\left(P_{m s}\right)$ increased bone formation, while the parameter $m_{b t}$ had the opposite effect. The production and denaturation of TGF$\beta$ had a very high influence on the TGF- $\beta$ concentration in the PDL. The same conclusion can be drawn for the concentration of RANKL in the PDL at the resorption site during tooth movement. This concentration was mainly influenced by the production and denaturation of RANKL. Decreasing the activity of osteoblasts or decreasing their life span speeds up bone resorption during tooth movement, as well as decreasing the half-life of TGF- $\beta$.

The results of all uniform designs were analysed using a Gaussian process with both a Gaussian and a cubic covariance function. The general trend of the results was the same, regardless of the applied covariance function. However, in some cases the cubic covariance function picked up on numerical errors, while the Gaussian covariance function was able to filter them out. On the other hand, when dealing with outliers, the cubic covariance function had less problems incorporating them into the analysis. When analysing the results of physical experiments outliers are usually the result of measurement errors and random noise, and incorporating them into the analysis should be avoided. When investigating computer models, such errors are not present, and outliers are indicators of abrupt changes in the outcome of the model. As such, they should not be ignored, but instead be investigated more closely.

With the results of the sensitivity analysis, it was possible to identify the ten parameters that had the highest influence on the outcome of the mechanobiological model presented in this work. Most notable was the high influence of osteoblast apoptosis and the half-life of TGF- $\beta$. When using experimental results to refine the estimates of the parameters, these are the parameters that should be looked at first. 
Some further observations were made that should be kept in mind when analysing and developing the model further. Some parameter combinations promoted bone resorption too strongly, resulting in a total loss of bone mass. Others resulted in a fast occurrence of a large number of osteoblasts in the bone. The effect of the mechanical stimulus on bone formation proved to be small, indicating a closer look into the assumption that osteoblasts will only form close to bone could be useful.

Some unexpected results did occur, and were investigated closer. In most cases valid explanations for the phenomenons were found, and no further actions were necessary, in other cases, suggestions for further analysis could be made. In summary, the extensive sensitivity analysis resulted in a better understanding of model and its parameters.

\section{Conclusions}

The design of experiments is a valuable tool for studying a large variety of computer models. Any type of model can be analysed using a DOE, as long as the experiment can be easily repeated using different parameters. The designs are developed to get as much information as possible at minimal cost, usually referring to the computational time. Each design has its pros and cons and depending on the needs of the researcher a suitable design is generated. Several statistical software packages provide support for DOE.

As with all statistical tools, care has to be taken when interpreting the results of a DOE, certainly with more complex models. Both the designs and the analysing methods have it limitations. However, keeping those in mind, DOE is a great tool, allowing the researcher to gain a better insight into their model and its behaviour in parameter space. Moreover, the results of a DOE analysis can be a good starting point for further model optimization (see Chapter 12 by Eriksson et al. elsewhere in this volume).

\section{Acknowledgements}

The authors acknowledge support from European Research Council under the European Union's Seventh Framework Programme (FP7/2007-2013)/ERC grant agreement n279100 (An Van Schepdael and Lies Geris). Aurélie Carlier is a Fellow of the Research Foundation Flanders (FWO).

\section{Conflict of interest}

The authors declare that they have no conflict of interest. 


\section{References}

1. A. Carlier, Y.C. Chai, M. Moesen, T. Theys, J. Schrooten, H. Van Oosterwyck, and L. Geris. Designing optimal calcium phosphate scaffold-cell combinations using an integrative modelbased approach. Acta Biomater, 7:3573-3585, 2011.

2. F.H. Dar, J.R. Meakin, and R.M. Aspden. Statistical methods in finite element analysis. $J$. Biomech., 35:1155-1161, 2002.

3. M. Ebden. Gaussian processes for regression: A quick introduction. Lecture notes, 2008.

4. K.T. Fang. The uniform design: application of number-theoretic methods in experimental design. Acta Mathematicae Applicatae Sinica, 3:363-372, 1980.

5. K.T. Fang, R. Li, and A. Sudijanto. Design and modeling for computer experiments. Chapman \& Hall/CRC, 2006.

6. R.A. Fisher. The Design of Experiments. Oliver \& Boyd, Edinburgh, 1935.

7. P. R. Garant, editor. Oral Cells and Tissues. Quintessence Publishing, 2003.

8. L. Geris, A. Gerisch, C. Maes, G. Carmeliet, R. Weiner, J. Vander Sloten, and H. Van Oosterwyck. Mathematical modeling of fracture healing in mice: comparison between experimental data and numerical simulation results. MBEC, 44:280-289, 2006.

9. Liesbet Geris, Alf Gerisch, Jos Vander Sloten, Rudiger Weiner, and Hans Van Oosterwyck. Angiogenesis in bone fracture healing: a bioregulatory model. J Theor Biol, 251(1):137-158, Mar 2008.

10. S. Henneman, J. W. Von den Hoff, and J. C. Maltha. Mechanobiology of tooth movement. Eur J Orthod, 30(3):299-306, Jun 2008.

11. Hanna Isaksson, Corrinus $C$ van Donkelaar, Rik Huiskes, and Keita Ito. A mechano-regulatory bone-healing model incorporating cell-phenotype specific activity. J Theor Biol, 252:230-246, 2008a.

12. Hanna Isaksson, Corrinus $C$ van Donkelaar, Rik Huiskes, Jiang Yao, and Keita Ito. Determining the most important cellular characteristics for fracture healing using design of experiments methods. J Theor Biol, 255(1):26-39, Nov 2008.

13. Hiroyuki Kanzaki, Mirei Chiba, Yoshinobu Shimizu, and Hideo Mitani. Periodontal ligament cells under mechanical stress induce osteoclastogenesis by receptor activator of nuclear factor kappab ligand up-regulation via prostaglandin e2 synthesis. J Bone Miner Res, 17(2):210-220, Feb 2002.

14. S. Kimoto, M. Matsuzawa, S. Matsubara, T. Komatsu, N. Uchimura, T. Kawase, and S. Saito. Cytokine secretion of periodontal ligament fibroblasts derived from human deciduous teeth: effect of mechanical stress on the secretion of transforming growth factor-beta 1 and macrophage colony stimulating factor. J Periodontal Res, 34(5):235-243, Jul 1999.

15. V. Krishnan and Z. Davidovitch. On a path to unfolding the biological mechanisms of orthodontic tooth movement. J Dent Res, 88(7):597-608, Jul 2009.

16. Vinod Krishnan and Ze'ev Davidovitch. Cellular, molecular, and tissue-level reactions to orthodontic force. Am J Orthod Dentofacial Orthop, 129(4):469.e1-469.32, Apr 2006.

17. D. Lacroix. Simulation of tissue differentiation during fracture healing. $\mathrm{PhD}$ thesis, University of Dublin, 2001.

18. C.L. Lin, S.H. Chang, W.J. Chang, and Kuo Y.C. Factorial analysis of variables influencing mechanical characteristics of a single tooth implant placed in the maxilla using finite element analysis and the statistics-based taguchi method. Eur J Oral Sci, 115:408-416, 2007.

19. T. Lundstedt, E. Seifert, L. Abramo, B. Thelin, A. Nyström, J. Pettersen, and R. Bergman. Experimental design and optimization. Chemometr Intell Lab, 42(2):3-40, Aug 1998.

20. D.J.C. MacKay. Introduction to gaussian processes. Lecture Notes, 1998.

21. A. Malandrino, J. Planell, and D. Lacroix. Statistical factorial analysis on the poroelastic material properties sensitivity of the lumbar intervertebral disc under compression, flexion and axial rotation. J Biomech, 42:2780-2788, 2009.

22. G. Marotti. The osteocyte as a wiring transmission system. J Musculoskelet Neuronal Interact, 1(2):133-136, Dec 2000. 
23. D.C. Montgomery. Design and analysis of experiments. John Wiley \& Sons, 7th edition, 1997.

24. R.H. Myers and D.C. Montgomery. Response surface methodology: process and product optimization using designed experiments. John Wiley \& Sons, 1995.

25. Y. Nishijima, M. Yamaguchi, T. Kojima, N. Aihara, R. Nakajima, and K. Kasai. Levels of rankl and opg in gingival crevicular fluid during orthodontic tooth movement and effect of compression force on releases from periodontal ligament cells in vitro. Orthod Craniofac Res, 9(2):63-70, May 2006.

26. J. Pfeilschifter, I. Diel, B. Scheppach, A. Bretz, R. Krempien, J. Erdmann, G. Schmid, N. Reske, H. Bismar, T. Seck, B. Krempien, and R. Ziegler. Concentration of transforming growth factor beta in human bone tissue: relationship to age, menopause, bone turnover, and bone volume. J Bone Miner Res, 13(4):716-730, Apr 1998.

27. Mark N Pinkerton, David C Wescott, Benjamin J Gaffey, Kyle T Beggs, Trudy J Milne, and Murray C Meikle. Cultured human periodontal ligament cells constitutively express multiple osteotropic cytokines and growth factors, several of which are responsive to mechanical deformation. J Periodontal Res, 43(3):343-351, Jun 2008.

28. Peter Pivonka, Jan Zimak, David W Smith, Bruce S Gardiner, Colin R Dunstan, Natalie A Sims, T. John Martin, and Gregory R Mundy. Model structure and control of bone remodeling: a theoretical study. Bone, 43(2):249-263, Aug 2008.

29. C.G. Provatidis. An analytical model for stress analysis of a tooth in translation. International Journal of Engineering Science, 39:1361-1381, 2001.

30. Daniel J Rinchuse, Donald J Rinchuse, Mark F Sosovicka, Janet M Robison, and Ryan Pendleton. Orthodontic treatment of patients using bisphosphonates: a report of 2 cases. Am J Orthod Dentofacial Orthop, 131(3):321-326, Mar 2007.

31. A. Saltelli, K. Chan, and E.M. Scott, editors. Sensitivity analysis. John Wiley \& Sons, 2000.

32. M. Sandberg, T. Vuorio, H. Hirvonen, K. Alitalo, and E. Vuorio. Enhanced expression of tgf-beta and c-fos mrnas in the growth plates of developing human long bones. Development, 102(3):461-470, Mar 1988.

33. T.J. Santner, B.J. Williams, and W.I. Notz. The design \& analysis of computer experiments. Springer-Verslag New York, 2003.

34. A. Van Schepdael, J. Vander Sloten, and L. Geris. A mechanobiological model of orthodontic tooth movement. Biomech Model Mechanobiol, 12(2):249-265, 2013.

35. A. Van Schepdael, J. Vander Sloten, and L. Geris. Mechanobiological modeling can explain orthodontic tooth movement: Three case studies. J. Biomech., 46(3):470-477, 2013.

36. D. C. Wescott, M. N. Pinkerton, B. J. Gaffey, K. T. Beggs, T. J. Milne, and M. C. Meikle. Osteogenic gene expression by human periodontal ligament cells under cyclic tension. J Dent Res, 86(12):1212-1216, Dec 2007.

37. M. Yamaguchi, N. Aihara, T. Kojima, and K. Kasai. Rankl increase in compressed periodontal ligament cells from root resorption. J Dent Res, 85(8):751-756, Aug 2006.

38. K. Yang, E.C. Teo, and F.K. Fuss. Application of taguchi method in optimization of cervical ring cage. J. Biomech., 40:3251-3256, 2007. 